\title{
“Inverse-Electron-Demand" Ligand Substitution: Experimental and Computational Insights into Olefin Exchange at Palladium(0)
}

\author{
Brian V. Popp, Joseph L. Thorman, Christine M. Morales, Clark R. Landis*, and \\ Shannon S. Stahl*
}

Department of Chemistry, University of Wisconsin-Madison, 1101 University Avenue, Madison, WI 53706

\section{Contents}

2D NMR Spectroscopic Characterization of $3^{\mathrm{CH}_{3}}$

Eyring Plots for Degenerate Olefin Exchange

Pseudo-first Order Olefin Dependence at Different Solvent Compositions

Gas Phase Geometry Optimized Isomers of $5 \bullet \mathrm{C}_{2} \mathrm{H}_{3} \mathrm{NO}_{2}$

Comparison of Experimental and Calculated Geometrical Parameters

Comparison of the Structural Properties of Calculated Olefin Substitution Species

Comparison of Basis Set Results for Ethylene Exchange Reaction

Gaussian98 Results (xyz coordinates, optimization gradients, total energy) for the Self-Exchange of $\mathbf{C}_{\mathbf{2}} \mathbf{H}_{\mathbf{4}}$ with $\mathbf{4}$ using the LANL2DZ basis set (Basis A).

Gaussian98 Results (xyz coordinates, optimization gradients, total energy) for the Self-Exchange of $\mathbf{C}_{2} \mathbf{H}_{3} \mathbf{N O}_{2}$ with $\mathbf{5}$ using the LANL2DZ basis set (Basis A).

Gaussian98 Results (xyz coordinates, optimization gradients, total energy) for the SelfExchange of $\mathbf{C}_{2} \mathbf{H}_{4}$ with 4 using the Stuttgart RSC 1997 ECP basis set (Basis B). $\quad$ S40-S48 
2D NMR Spectroscopic Characterization of $\mathbf{3}^{\mathbf{C H}_{3}}$. Advanced two-dimensional NMR spectroscopic pulse programs were employed on a Varian INOVA 500 to identify the positions of the olefinic protons bound to palladium. The first method, gradient selected phase sensitive Heteronuclear Single Quantum Correlation (gHSQC) spectroscopy, was used to identify the chemical shifts of all carbon nuclei that have a ${ }^{1} \mathrm{~J}_{\mathrm{CH}}$ coupling (Figure S1 and Table S1). VNMR 6.1 parameters used for the acquisition: $a t=0.318 \mathrm{~s}, \mathrm{nt}=2, \mathrm{ss}=32, \mathrm{~d} 1=5.0 \mathrm{~s}, \mathrm{ni}=400, \mathrm{j} 1 \times \mathrm{xh}=140.0$, temp $=24^{\circ} \mathrm{C}$. The second method, gradient selected absolute value Heteronuclear Multiple-Bond Correlation (gHMBC) spectroscopy, was used to identify the chemical shifts of carbon nuclei that have either a ${ }^{2} \mathrm{~J}_{\mathrm{CH}}$ or ${ }^{3} \mathrm{~J}_{\mathrm{CH}}$ coupling (Figure S2 and Table S2). VNMR 6.1 software parameters used for the acquisition: at $=0.318 \mathrm{~s}, \mathrm{nt}=8, \mathrm{ss}=32, \mathrm{~d} 1=5.0 \mathrm{~s}, \mathrm{ni}=400, \mathrm{~J} 1 \times \mathrm{xh}=140.0, \mathrm{Jn} \times \mathrm{Xh}=8.0$, temp $=24.0^{\circ} \mathrm{C}$. 


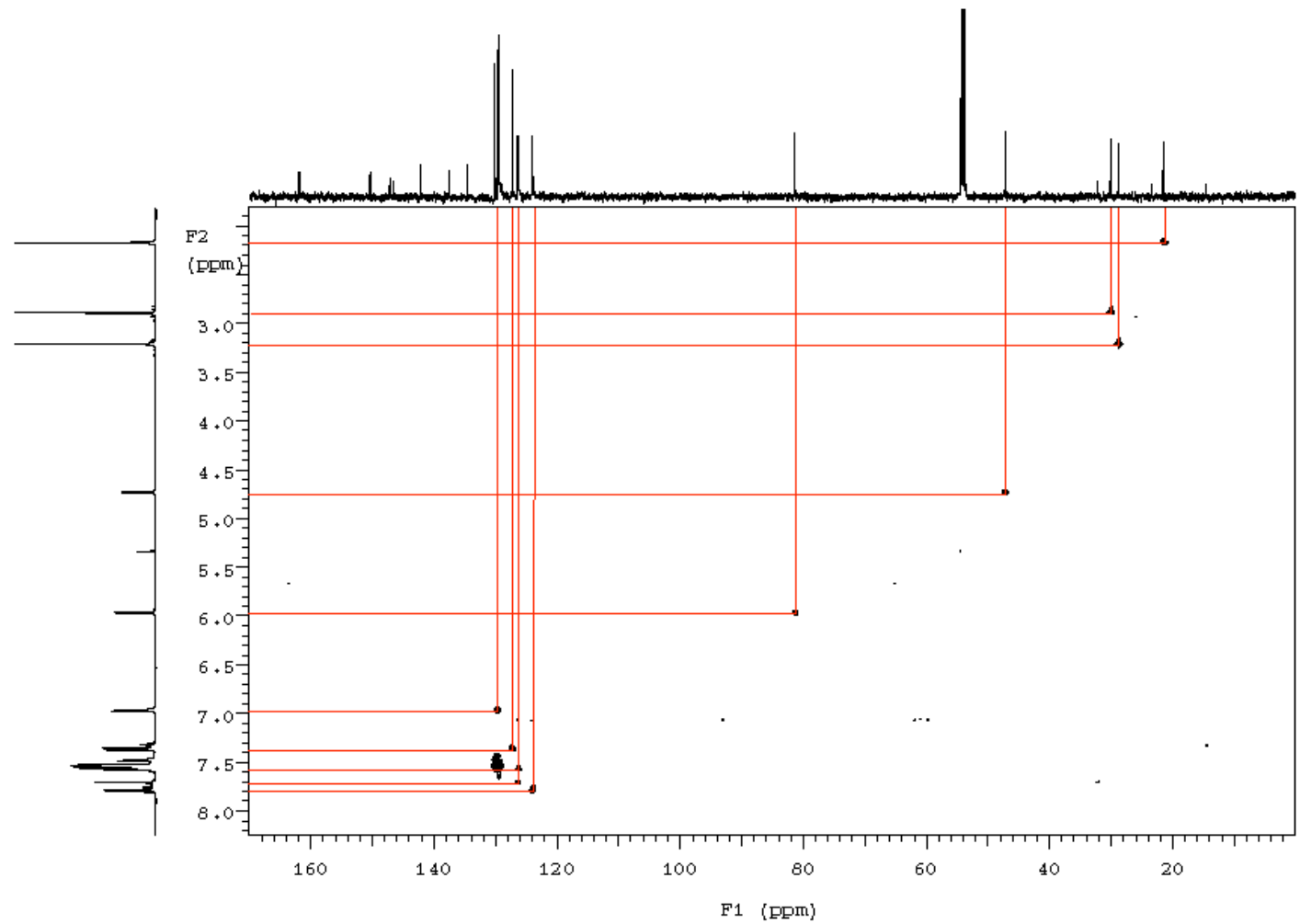

Figure S1. gHSQC of $3^{\mathrm{CH} 3}$.

Table S1. ${ }^{1} \mathrm{~J}_{\mathrm{CH}}$ Correlation Table

$\begin{array}{ccc}\delta_{1 \mathrm{H}}(\mathrm{ppm}) & \text { Assignment } & \text { HSQC Correlation }\left(\delta_{13 \mathrm{C}} \mathrm{ppm}\right) \\ 2.167 & \mathrm{a} & 21.46 \\ 2.888 & \mathrm{~b} & 29.97 \\ 3.206 & \mathrm{c} & 28.72 \\ 4.729 & \mathrm{~d} & 47.14 \\ 5.963 & \mathrm{e} & 81.29 \\ 6.964 & \mathrm{f} & 129.53 \\ 7.361 & \mathrm{~g} & 127.15 \\ 7.500 & \mathrm{~h} & 129.29 / 129.32 / 129.58 / 129.97 / 130.18 \\ 7.581 & \mathrm{i} & 126.12 \\ 7.703 & \mathrm{j} & 126.34 \\ 7.766 & \mathrm{k} & 123.85 \\ 7.798 & \mathrm{l} & 123.93\end{array}$

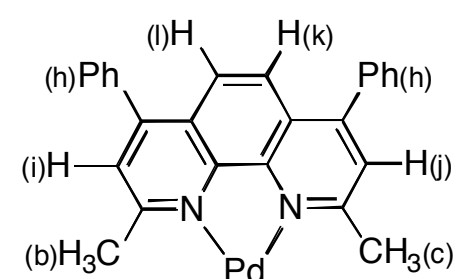

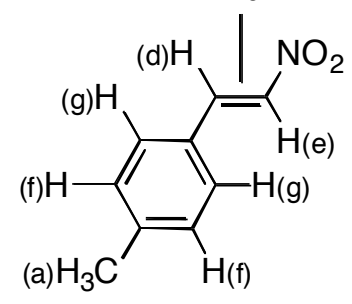




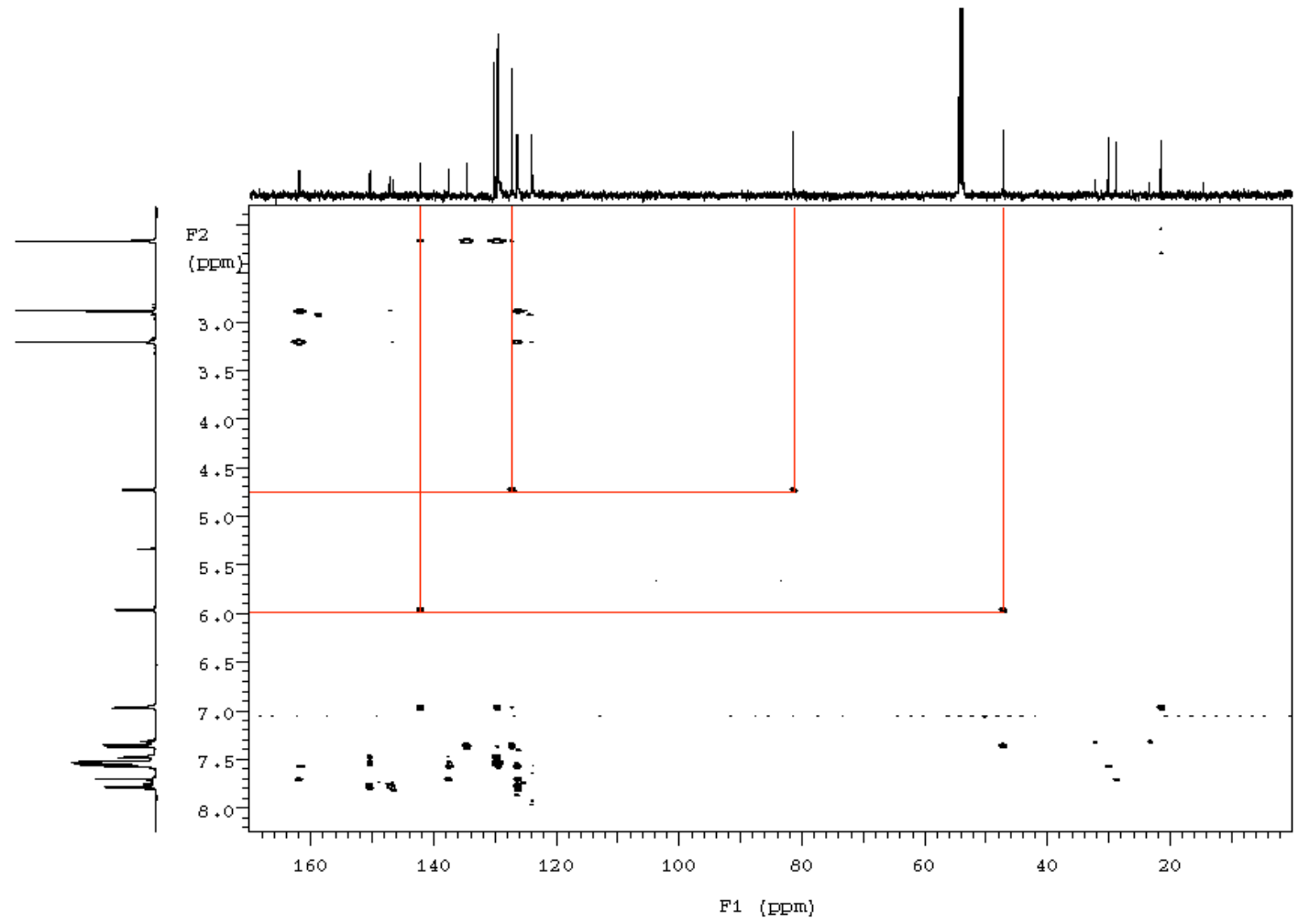

Figure S2. gHMBC of $\mathbf{3}^{\mathrm{CH} 3}$. 
Table S2. ${ }^{2} \mathbf{J}_{\mathrm{CH}}$ and ${ }^{3} \mathrm{~J}_{\mathrm{CH}}$ Correlation Table

\begin{tabular}{ccl}
$\delta_{1 \mathrm{H}}(\mathrm{ppm})$ & Assignment & \multicolumn{1}{c}{ HMBC Correlation $\left(\delta_{13 \mathrm{C}} \mathrm{ppm}, \mathrm{J}_{\mathrm{CH}}=2\right.$ or 3, intensity $)$} \\
2.167 & $\mathrm{a}$ & $(129.531,3 \mathrm{~J}, \mathrm{~s})(134.508,2 \mathrm{~J}, \mathrm{~s})$ \\
2.888 & $\mathrm{~b}$ & $(126.124,3 \mathrm{~J}, \mathrm{~m})(161.624,2 \mathrm{~J}, \mathrm{~m})$ \\
3.206 & $\mathrm{c}$ & $(126.339,3 \mathrm{~J}, \mathrm{~m})(161.843,2 \mathrm{~J}, \mathrm{~m})$ \\
4.729 & $\mathrm{~d}$ & $(81.292,2 \mathrm{~J}, \mathrm{~s})(127.152,3 \mathrm{~J}, \mathrm{~m})$ \\
5.963 & $\mathrm{e}$ & $(47.137,2 \mathrm{~J}, \mathrm{~s})(142.089,3 \mathrm{~J}, \mathrm{~m})$ \\
6.964 & $\mathrm{f}$ & $(21.459,3 \mathrm{~J}, \mathrm{~m})(129.531,3 \mathrm{~J}, \mathrm{~s})(142.089,3 \mathrm{~J}, \mathrm{vs})$ \\
7.361 & $\mathrm{~g}$ & $(47.137,3 \mathrm{~J}, \mathrm{~s})(127.152,3 \mathrm{~J}, \mathrm{~s})(134.508,3 \mathrm{~J}, \mathrm{vs})$ \\
7.500 & $\mathrm{~h}$ & $($ unassignable $129-130)(150.261, \mathrm{vw})(150.382, \mathrm{vw})$ \\
7.581 & $\mathrm{i}$ & $(29.971,3 \mathrm{~J}, \mathrm{w})(126.205, \mathrm{w})(137.410, \mathrm{w})(161.624, \mathrm{w})$ \\
7.703 & $\mathrm{j}$ & $(28.720,3 \mathrm{~J}, \mathrm{w})(126.248, \mathrm{w})(137.508, \mathrm{w})(161.843, \mathrm{w})$ \\
7.766 & $\mathrm{k}$ & $(126.248,3 \mathrm{~J}, \mathrm{w})(147.107,3 \mathrm{~J}, \mathrm{~m})(150.382,3 \mathrm{~J}, \mathrm{w})$ \\
7.798 & $\mathrm{l}$ & $(126.205,3 \mathrm{~J}, \mathrm{w})(146.545,3 \mathrm{~J}, \mathrm{~m})(150.261,3 \mathrm{~J}, \mathrm{w})$ \\
& &
\end{tabular}

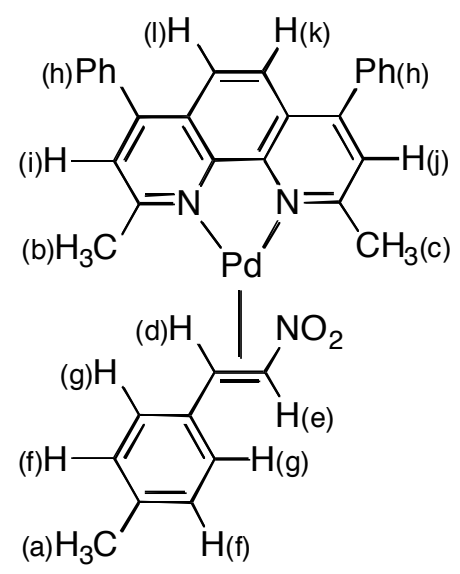




\section{Eyring Plots for Degenerate Olefin Exchange}

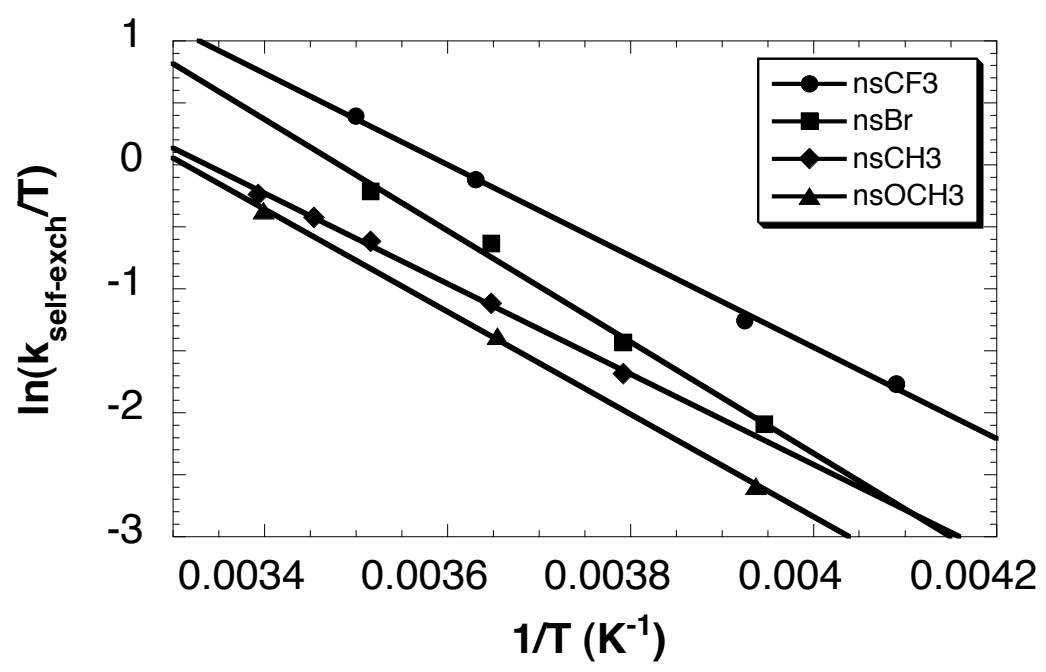

Figure S3. Eyring plots showing the temperature dependent behavior of the degenerate exchange of olefin between $3^{\mathrm{x}}$ and $\mathrm{ns}^{\mathrm{x}}$. Reaction conditions: $[\mathrm{Pd}]=10 \mathrm{mM},\left[\mathrm{ns}^{\mathrm{X}}\right]=60 \mathrm{mM}, \mathrm{CD}_{2} \mathrm{Cl}_{2}$. 


\section{Pseudo-First-Order Olefin Dependence at Different Solvent Compositions}

A.

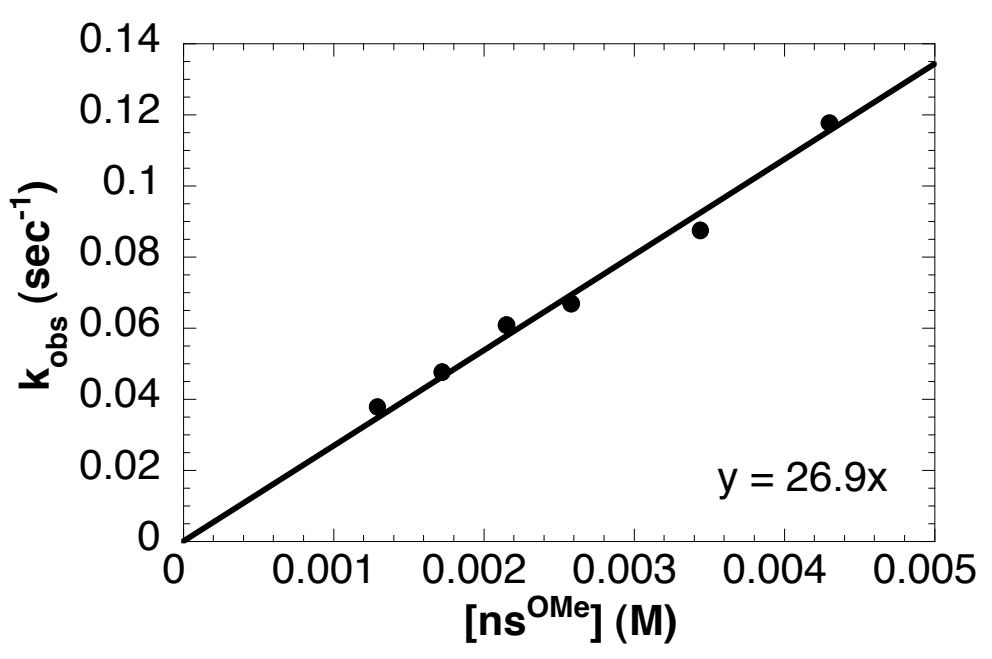

B.

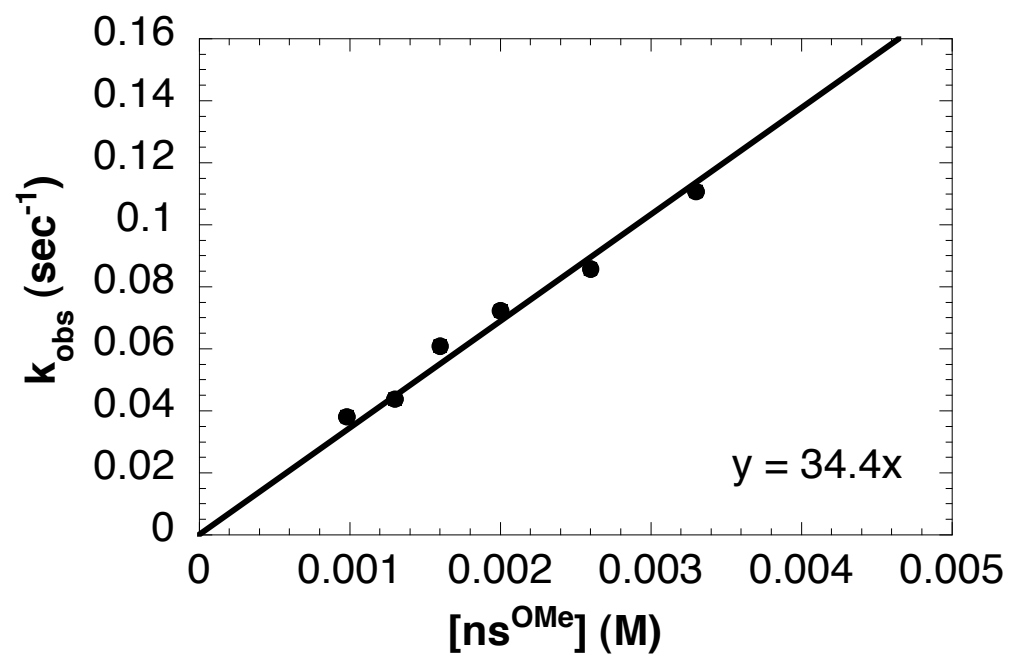

Figure S4. Pseudo-first order olefin concentration dependence for the associative ligand substitution of the $\mathrm{ns}^{\mathrm{CH}_{3}}$ of $\mathbf{3}^{\mathrm{CH3}}$ for $\mathrm{ns}^{\mathrm{OCH}_{3}}$. Observed rate measured by changes in the UV-vis spectrum at $445 \mathrm{~nm}$. Reaction conditions in $\mathrm{A}:[\mathrm{Pd}]=110 \mathrm{uM}$, [nsOMe] $=1.3-4.3 \mathrm{mM}, 1.5 \mathrm{ml}$ $\mathrm{CH}_{2} \mathrm{Cl}_{2}$ and $1.5 \mathrm{ml}$ Toluene, $298 \mathrm{~K}$. Reaction conditions in $\mathrm{B}$ : $[\mathrm{Pd}]=110 \mathrm{uM},[\mathrm{nsOMe}]=1.0-$ $3.9 \mathrm{mM}, 0.2 \mathrm{ml} \mathrm{CH}_{2} \mathrm{Cl}_{2}$ and $2.8 \mathrm{ml}$ Toluene, $298 \mathrm{~K}$. 
Gas Phase Geometry Optimized Isomers of $5 \cdot \mathrm{C}_{2} \mathrm{H}_{3} \mathrm{NO}_{2}$

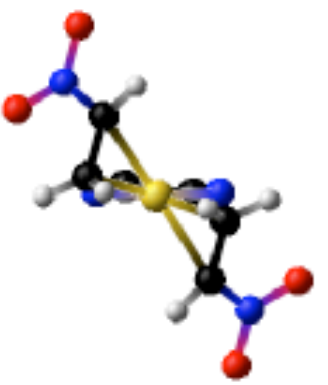

$\left[5 \cdot \mathrm{C}_{2} \mathrm{H}_{3} \mathrm{NO}\right]-\mathrm{A}$

$E_{\mathrm{re} \mid}=0 \mathrm{kcal} / \mathrm{mol}$

$\mu=0.73$ debeye

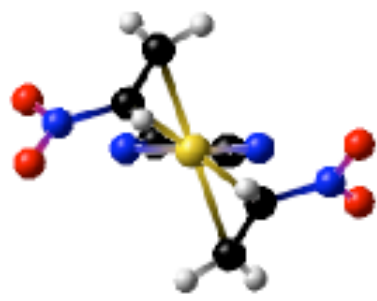

$\left[5 \cdot \mathrm{C}_{2} \mathrm{H}_{3} \mathrm{NO}\right] \mathrm{E}$

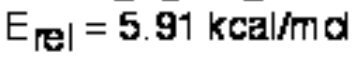
$\mu=9.37$ debeye

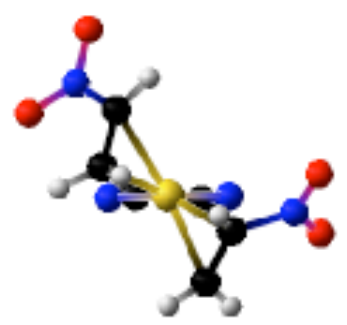

$\left.\mathbf{E} \cdot \mathrm{C}_{2} \mathrm{H}_{3} \mathrm{~N} \boldsymbol{Q}_{2}\right]-\mathrm{B}$ $E_{r e l}=2.35 \mathrm{kcal} / \mathrm{md}$ $\mu=5.97$ debeye

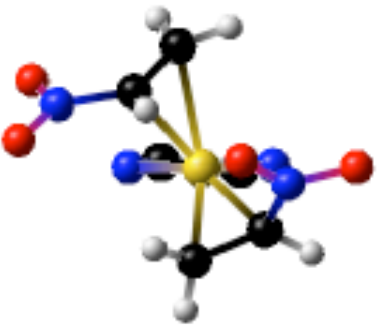

Б. $\left.\mathrm{C}_{2} \mathrm{H}_{3} \mathrm{NO}\right]-\mathrm{F}$ $E_{r e l}=7.32 \mathrm{kcal} / \mathrm{md}$ $\mu=\mathbf{0 . 9 9}$ debeye

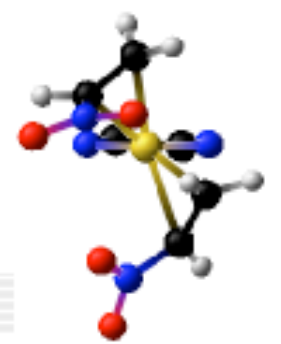

$\left[5+\mathrm{C}_{2} \mathrm{H}_{3} \mathrm{NO}\right]-\mathrm{I}$

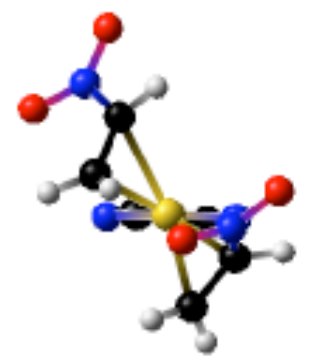

Б. $\left.\mathrm{C}_{2} \mathrm{H}_{3} \mathrm{NQ}\right]-\mathrm{C}$ $E_{r e l}=\mathbf{5 . 9 1} \mathrm{kcal} / \mathrm{mo}$ $\mu=9.37$ debeye

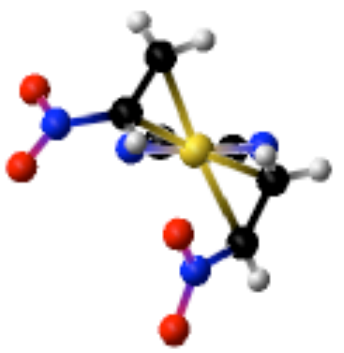

$\left[5 \cdot \mathrm{C}_{2} \mathrm{H}_{3} \mathrm{NO}\right]-\mathrm{G}$

$E_{r e l}=4.52 \mathrm{kcal} / \mathrm{m} \mathrm{d}$ $\mu=5.01$ debeye

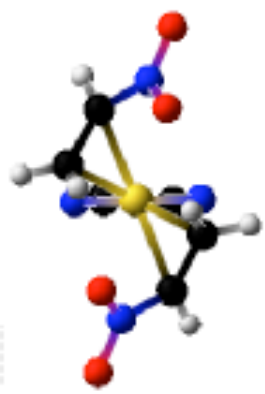

$\left[5 \cdot \mathrm{C}_{2} \mathrm{H}_{3} \mathrm{NO}\right]$

$E_{r e l}=13.15 \mathrm{kcal} / \mathrm{mod}$

$E_{\text {rel }}=5.64 \mathrm{kcal} / \mathrm{ml}$ $\mu=13.13$ debeye $\mu=10.72$ debeye

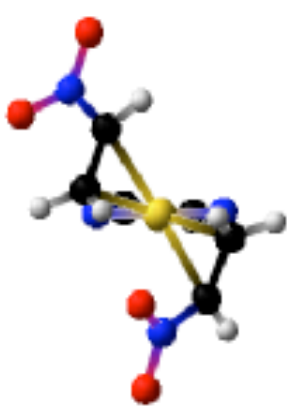

$\left.\bar{b} \cdot \mathrm{C}_{2} \mathrm{H}_{3} \mathrm{~N} \boldsymbol{O}_{2}\right]-\mathrm{D}$ $E_{\mathrm{rel}}=2.59 \mathrm{kcal} / \mathrm{md}$ $\mu=\mathbf{8 . 2 9}$ debeye

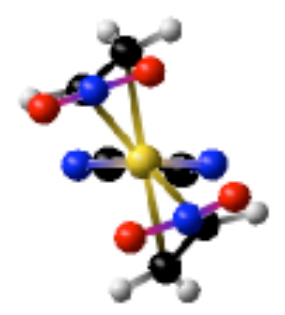

Б* $\left.\mathrm{C}_{2} \mathrm{H}_{3} \mathrm{NO}\right]-\mathrm{H}$

$E_{r e l}=21.45 \mathrm{kcal} / \mathrm{mol}$ $\mu=15.03$ debeye

Figure S5. Ball-and-stick models of the different possible conformation of (di) $\mathrm{Pd}\left(\mathrm{C}_{2} \mathrm{H}_{3} \mathrm{NO}_{2}\right)_{2}$ $\left(\mathbf{5} \cdot \mathbf{C}_{2} \mathbf{H}_{4} \mathbf{N O}_{2}\right)$ together with their calculated relative energies and molecular dipole moments. Isomers $-\mathrm{B},-\mathrm{C}$, and $-\mathrm{F}$ did not optimize fully. 


\section{Comparison of Experimental and Calculated Geometrical Parameters}

Table S3. Bond Length and Angle Comparison [ $\AA^{\circ}$ and $\left.{ }^{\circ}\right]$.

\begin{tabular}{|c|c|c|c|c|c|}
\hline & $\mathbf{3}^{\mathbf{H}}$ (X-ray) & $4\left(\mathrm{DFT}^{\mathrm{a}}\right)$ & $5\left(\mathrm{DFT}^{\mathrm{a}}\right)$ & & \\
\hline Pd-N1 & $2.156(7)$ & 2.19 & 2.19 & & \\
\hline $\mathrm{Pd}-\mathrm{N} 2$ & $2.161(8)$ & 2.19 & 2.16 & & \\
\hline $\mathrm{Pd}-\mathrm{C} 1$ & $2.040(10)$ & 2.14 & 2.13 & & \\
\hline $\mathrm{Pd}-\mathrm{C} 2$ & $2.075(9)$ & 2.14 & 2.11 & & $R$ \\
\hline $\mathrm{C} 1-\mathrm{C} 2$ & $1.427(14)$ & 1.43 & 1.44 & $x$ & \\
\hline $\mathrm{N} 1-\mathrm{Pd}-\mathrm{N} 2$ & $76.8(3)$ & 73.8 & 74.5 & \multirow[t]{2}{*}{$\mathrm{X}=\mathrm{NO}_{2}, \mathrm{H}$} & $\mathrm{R}=\mathrm{H}, \mathrm{Ph}$ \\
\hline $\mathrm{C} 1-\mathrm{Pd}-\mathrm{C} 2$ & $40.6(4)$ & 39.2 & 39.5 & & \\
\hline
\end{tabular}

${ }^{a}$ The DFT calculations were performed using the B3LYP functional with a LANL2DZ basis set which includes a relativistic effective core potential applied to the $\mathrm{Pd}$ atom. 
Comparison of the Structural Parameters of the Calculated Structures in the Olefin Substitution Reactions.

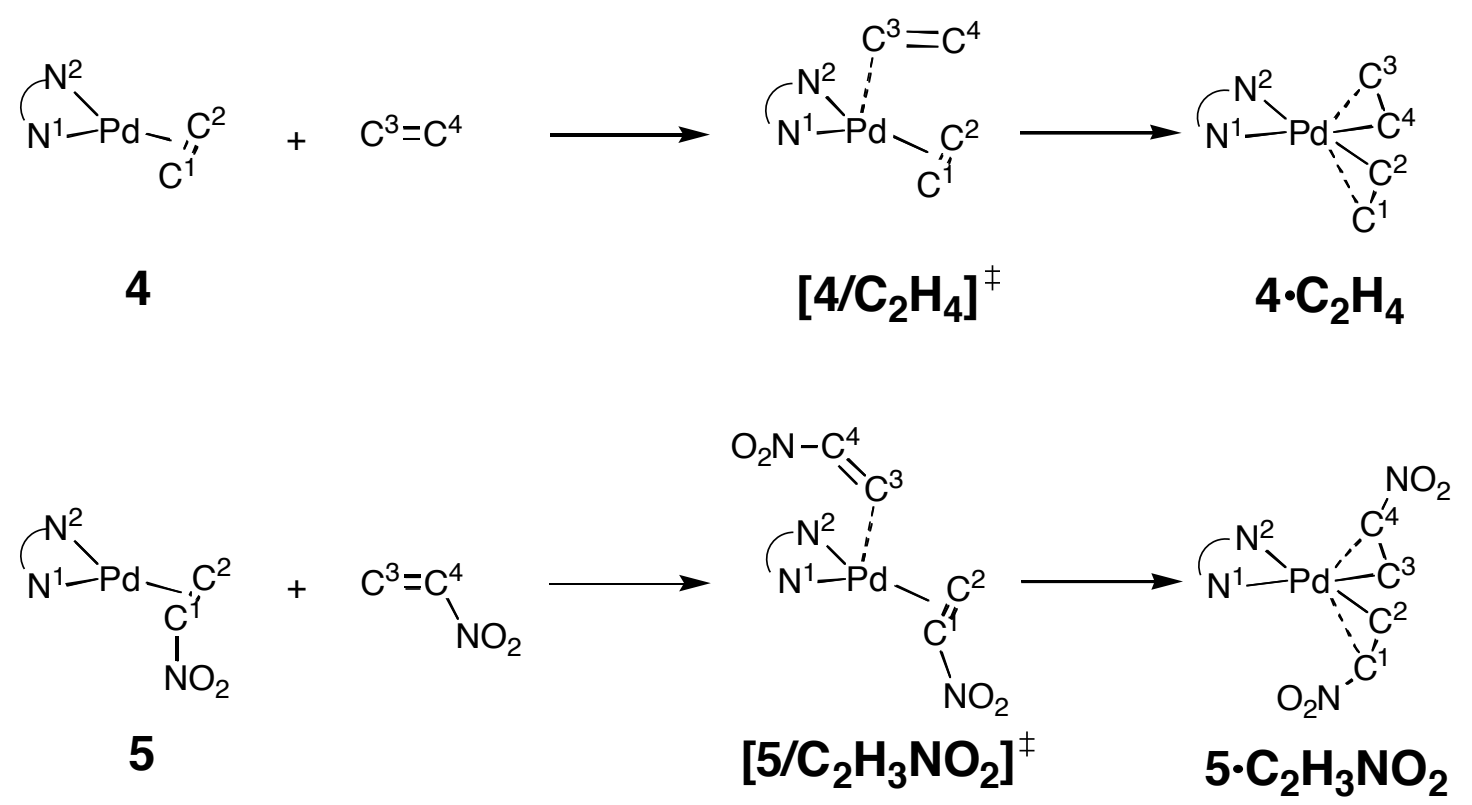

Table S4. Structural Properties of Olefin Substitution Species $\left[\AA\right.$ and $\left.^{\circ}\right] .^{\mathrm{a}}$

\begin{tabular}{lcccccc} 
& $\mathbf{4}$ & {$\left[\mathbf{4} / \mathbf{C}_{2} \mathbf{H}_{\mathbf{4}}\right]^{\ddagger}$} & $\mathbf{4} \cdot \mathbf{C}_{2} \mathbf{H}_{\mathbf{4}}$ & $\mathbf{5}$ & {$\left[\mathbf{5} / \mathbf{C}_{2} \mathbf{H}_{3} \mathbf{N} \mathbf{O}_{2}\right]^{\ddagger}$} & $\mathbf{5} \cdot \mathbf{C}_{2} \mathbf{H}_{3} \mathbf{N} \mathbf{O}_{2}$ \\
\hline Pd-N1 & 2.19 & 2.17 & 2.12 & 2.19 & 2.22 & 2.20 \\
Pd-N2 & 2.19 & 2.16 & 2.12 & 2.16 & 2.28 & 2.20 \\
Pd-C1 & 2.14 & 2.19 & 2.32 & 2.13 & 2.17 & 2.32 \\
Pd-C2 & 2.14 & 2.16 & 2.23 & 2.11 & 2.13 & 2.15 \\
Pd-C3 & NA & 2.91 & 2.32 & NA & 2.31 & 2.15 \\
Pd-C4 & NA & 3.08 & 2.23 & NA & 2.60 & 2.32 \\
C1-C2 & 1.43 & 1.42 & 1.40 & 1.44 & 1.42 & 1.42 \\
C3-C4 & 1.35 & 1.35 & 1.40 & 1.34 & 1.39 & 1.42 \\
N1-Pd-N2 & 73.8 & 75.3 & 76.9 & 74.5 & 73.4 & 74.4 \\
Pd-C1-C2 & 70.4 & 69.6 & 68.3 & 69.4 & 69.2 & 64.8 \\
Pd-C2-C1 & 70.4 & 72.2 & 75.9 & 71.1 & 72.2 & 78.5 \\
Pd-C3-C4 & NA & 83.9 & 68.3 & NA & 85.6 & 78.5 \\
Pd-C4-C3 & NA & 70.2 & 75.9 & NA & 62.3 & 64.8 \\
\hline
\end{tabular}

${ }^{\mathrm{a}}$ Gas phase geometry optimization calculations were performed using a LANL2DZ basis set. 
Comparison of Basis Sets. Two different basis sets were used in the calculation of the degenerate ethylene exchange reaction: LANL2DZ (BASIS- $A$ ) and Stuttgart RSC 1997 ECP $(B A S I S-B)$. Basis- $B$ is a comparatively larger basis set which utilizes a triple- $\zeta$ basis set with diffuse and polarization functions $\left(6-311++\mathrm{G}^{* *}\right)$ added to $\mathrm{C}, \mathrm{N}$, and $\mathrm{H}$. All geometry optimization calculations were independently performed in both basis sets. The optimized geometries show little change upon increasing the basis set size (Table S5). The relative energies of the structures calculated with the two basis sets were also compared (Table S6). Because of the relatively small differences observed with the higher level basis set $(B A S I S-B)$ together with its substantially higher cost, most of the calculations in this study were performed with the LANL2DZ basis set.

Table S5. Basis Set Effects on the Gas-Phase Geometrical Parameters [ $\AA$ and ${ }^{\circ}$ ] Optimized in the Degenerate Ethylene Exchange Reaction.

\begin{tabular}{|c|c|c|c|c|c|c|}
\hline \multirow[t]{3}{*}{$\begin{array}{c}\mathrm{N}^{1}-\mathrm{Pd}-\mathrm{C}^{2} \\
\mathrm{C}^{1} \\
4\end{array}$} & + & $c^{3}=c^{4}$ & $\rightarrow$ & {$[4 /$} & $\begin{array}{l}=\mathrm{C}^{4} \\
\mathrm{C}^{\mathrm{C}^{2}} \\
\left.{ }_{2} \mathrm{H}_{4}\right]^{\mp}\end{array}$ & \\
\hline & & Basis-A & & & Basis-B & \\
\hline & 4 & {$\left[4 / \mathrm{C}_{2} \mathrm{H}_{4}\right]^{*}$} & $4 \cdot \mathrm{C}_{2} \mathrm{H}_{4}$ & 4 & {$\left[4 / \mathrm{C}_{2} \mathrm{H}_{4}\right]^{\frac{1}{t}}$} & $4 \cdot \mathrm{C}_{2} \mathrm{H}_{4}$ \\
\hline Pd-N1 & 2.19 & 2.17 & 2.12 & 2.23 & 2.17 & 2.14 \\
\hline Pd-N2 & 2.19 & 2.16 & 2.12 & 2.23 & 2.16 & 2.14 \\
\hline $\mathrm{Pd}-\mathrm{C} 1$ & 2.14 & 2.19 & 2.32 & 2.11 & 2.19 & 2.28 \\
\hline $\mathrm{Pd}-\mathrm{C} 2$ & 2.14 & 2.16 & 2.23 & 2.11 & 2.16 & 2.19 \\
\hline Pd-C3 & NA & 2.91 & 2.32 & NA & 2.91 & 2.28 \\
\hline $\mathrm{Pd}-\mathrm{C} 4$ & NA & 3.08 & 2.23 & NA & 3.08 & 2.19 \\
\hline $\mathrm{C} 1-\mathrm{C} 2$ & 1.43 & 1.42 & 1.40 & 1.41 & 1.42 & 1.39 \\
\hline $\mathrm{C} 3-\mathrm{C} 4$ & 1.35 & 1.35 & 1.40 & 1.33 & 1.35 & 1.39 \\
\hline $\mathrm{N} 1-\mathrm{Pd}-\mathrm{N} 2$ & 73.8 & 75.3 & 76.9 & 72.3 & 75.3 & 75.6 \\
\hline $\mathrm{Pd}-\mathrm{C} 1-\mathrm{C} 2$ & 70.4 & 69.6 & 68.3 & 70.5 & 69.6 & 68.2 \\
\hline $\mathrm{Pd}-\mathrm{C} 3-\mathrm{C} 4$ & NA & 83.9 & 68.3 & NA & 83.9 & 68.2 \\
\hline
\end{tabular}


Table S6. Basis-Set Effects on the Relative Energies of Structures in the Degenerate Ethylene Exchange Reaction. ${ }^{\text {a }}$

\begin{tabular}{lcccccc}
\hline & \multicolumn{3}{c}{ Basis- $A$} & \multicolumn{3}{c}{ Basis-B } \\
\hline & $\mathbf{4}$ & {$\left[\mathbf{4} / \mathbf{C}_{\mathbf{2}} \mathbf{H}_{\mathbf{4}}\right]^{\ddagger}$} & $\mathbf{4} \cdot \mathbf{C}_{\mathbf{2}} \mathbf{H}_{\mathbf{4}}$ & $\mathbf{4}$ & {$\left[\mathbf{4} / \mathbf{C}_{\mathbf{2}} \mathbf{H}_{\mathbf{4}}\right]^{\ddagger}$} & $\mathbf{4} \cdot \mathbf{C}_{\mathbf{2}} \mathbf{H}_{\mathbf{4}}$ \\
$\mathrm{G}_{\mathrm{CH} 2 \mathrm{C} 12}$ & 0 & 16.3 & 11.2 & 0 & 16.4 & 13.2 \\
$\mathrm{G}_{\text {Toluene }}$ & 0 & 14.7 & 11.0 & 0 & 15.1 & 11.3 \\
\hline
\end{tabular}

${ }^{\mathrm{a}}$ Energies are listed in $\mathrm{kcal} / \mathrm{mol}$. 
Gaussian98 Results (xyz cooridinates, optimization gradients, total energy) for the SelfExchange of $\mathrm{C}_{2} \mathrm{H}_{4}$ with 4 using the LANL2DZ basis set (Basis $A$ ).

\section{4-gas phase}

\begin{tabular}{|c|c|c|c|c|c|}
\hline \multirow{2}{*}{$\begin{array}{l}\text { Center } \\
\text { Number }\end{array}$} & \multirow{2}{*}{\multicolumn{2}{|c|}{$\begin{array}{l}\text { Atomic } \\
\text { Number }\end{array}$}} & \multirow{2}{*}{$\begin{array}{l}\text { Atomic } \\
\text { Type }\end{array}$} & \multicolumn{2}{|c|}{ Coordinates (Angstroms } \\
\hline & & & & $\mathrm{X}$ & $\mathrm{Z}$ \\
\hline 1 & 46 & 0 & 0.412055 & -0.000476 & -0.062003 \\
\hline 2 & 7 & 0 & -1.341218 & 1.316388 & -0.029238 \\
\hline 3 & 6 & 0 & -2.509793 & 0.734818 & 0.090403 \\
\hline 4 & 6 & 0 & -2.510549 & -0.733547 & 0.088249 \\
\hline 5 & 7 & 0 & -1.342226 & -1.316042 & -0.029137 \\
\hline 6 & 1 & 0 & -1.345087 & 2.341523 & -0.041014 \\
\hline 7 & 1 & 0 & -3.455960 & 1.271674 & 0.185365 \\
\hline 8 & 1 & 0 & -3.457621 & -1.269681 & 0.178159 \\
\hline 9 & 1 & 0 & -1.347313 & -2.341116 & -0.045094 \\
\hline 10 & 6 & 0 & 2.418119 & 0.716998 & 0.112895 \\
\hline 11 & 6 & 0 & 2.418559 & -0.715870 & 0.117674 \\
\hline 12 & 1 & 0 & 2.710046 & 1.259261 & -0.787212 \\
\hline 13 & 1 & 0 & 2.558048 & 1.268217 & 1.042820 \\
\hline 14 & 1 & 0 & 2.710745 & -1.263957 & -0.778825 \\
\hline 15 & 1 & 0 & 2.558681 & -1.260851 & 1.051227 \\
\hline
\end{tabular}

$\begin{array}{llll}\text { Maximum Force } & 0.000004 & 0.000450 & \text { YES } \\ \text { RMS Force } & 0.000002 & 0.000300 & \text { YES } \\ \text { Maximum Displacement } & 0.001904 & 0.001800 & \text { NO } \\ \text { RMS Displacement } & 0.000451 & 0.001200 & \text { YES } \\ \text { Predicted change in Energy=-2.665730D-09 } & \end{array}$

SCF Done: $\mathrm{E}(\mathrm{RB}+\mathrm{HF}-\mathrm{LYP})=-393.421588345 \quad$ A.U. after 2 cycles Convg $=0.3969 \mathrm{D}-08 \quad \mathrm{~S} * * 2=0.0000$

\section{$\underline{\mathbf{C}}_{2} \underline{H}_{4}$ - gas phase}

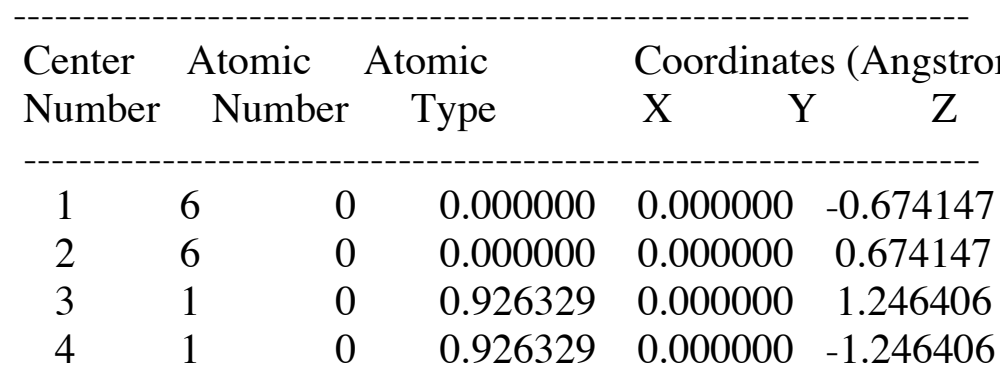




$\begin{array}{rrrrrr}5 & 1 & 0 & -0.926329 & 0.000000 & 1.246406 \\ 6 & 1 & 0 & -0.926329 & 0.000000 & -1.246406\end{array}$

$\begin{array}{lccc}\text { Maximum Force } & 0.000289 & 0.000450 & \text { YES } \\ \text { RMS Force } & 0.000170 & 0.000300 & \text { YES } \\ \text { Maximum Displacement } & 0.001337 & 0.001800 & \text { YES } \\ \text { RMS Displacement } & 0.000824 & 0.001200 & \text { YES } \\ \text { Predicted change in Energy=-7.602817D-07 } & \end{array}$

SCF Done: $\mathrm{E}(\mathrm{RB}+\mathrm{HF}-\mathrm{LYP})=-78.5782080480 \quad$ A.U. after 1 cycles

$$
\text { Convg }=0.9046 \mathrm{D}-10 \quad \mathrm{~S} * * 2=0.0000
$$

\section{$\left[4 / \mathrm{C}_{2} \underline{\mathbf{H}_{4}}\right]^{*}$ - gas phase}

\begin{tabular}{|c|c|c|c|c|c|}
\hline \multirow{2}{*}{$\begin{array}{l}\text { Center } \\
\text { Number }\end{array}$} & \multirow{2}{*}{\multicolumn{2}{|c|}{$\begin{array}{l}\text { Atomic } \\
\text { Number }\end{array}$}} & \multirow{2}{*}{$\begin{array}{r}\text { Atomic } \\
\text { Type }\end{array}$} & \multicolumn{2}{|c|}{ Coordinates (Angstrom } \\
\hline & & & & X $\quad \mathrm{Y}$ & $\mathrm{Z}$ \\
\hline 1 & 46 & 0 & 0.103190 & 0.253604 & -0.173043 \\
\hline 2 & 6 & 0 & 0.118852 & 0.315960 & 2.739618 \\
\hline 3 & 6 & 0 & 1.454557 & 0.154830 & 2.591232 \\
\hline 4 & 1 & 0 & -0.556534 & -0.533138 & 2.812714 \\
\hline 5 & 1 & 0 & -0.332953 & 1.302548 & 2.811378 \\
\hline 6 & 1 & 0 & 1.909425 & -0.832124 & 2.536866 \\
\hline 7 & 1 & 0 & 2.129633 & 1.005490 & 2.529987 \\
\hline 8 & 7 & 0 & 0.330278 & -1.898181 & -0.363291 \\
\hline 9 & 6 & 0 & -0.735848 & -2.529515 & -0.838119 \\
\hline 10 & 6 & 0 & -1.858128 & -1.703968 & -1.225183 \\
\hline 11 & 7 & 0 & -1.841153 & -0.448865 & -0.783713 \\
\hline 12 & 6 & 0 & 1.481261 & 1.837052 & -0.799075 \\
\hline 13 & 6 & 0 & 0.208535 & 2.386373 & -0.479478 \\
\hline 14 & 1 & 0 & 1.075919 & -2.507597 & -0.008875 \\
\hline 15 & 1 & 0 & -0.798578 & -3.615110 & -0.931009 \\
\hline 16 & 1 & 0 & -2.648555 & -2.111791 & -1.856966 \\
\hline 17 & 1 & 0 & -2.620874 & 0.137277 & -1.101763 \\
\hline 18 & 1 & 0 & 2.306852 & 1.908937 & -0.092868 \\
\hline 19 & 1 & 0 & 1.767882 & 1.663709 & -1.834385 \\
\hline 20 & 1 & & 0.059544 & 2.908087 & 0.465808 \\
\hline 21 & 1 & 0 & -0.477730 & 2.672851 & -1.275826 \\
\hline
\end{tabular}

$\begin{array}{llll}\text { Maximum Force } & 0.000006 & 0.000450 & \text { YES } \\ \text { RMS Force } & 0.000002 & 0.000300 & \text { YES } \\ \text { Maximum Displacement } & 0.001183 & 0.001800 & \text { YES } \\ \text { RMS Displacement } & 0.000251 & 0.001200 & \text { YES }\end{array}$


Predicted change in Energy=-8.436333D-09

SCF Done: $\mathrm{E}(\mathrm{RB}+\mathrm{HF}-\mathrm{LYP})=-471.996691772$ A.U. after 2 cycles Convg $=0.2245 \mathrm{D}-08 \quad \mathrm{~S} * * 2=0.0000$

\section{$\underline{4 \cdot \mathbf{C}_{2}} \underline{\mathrm{H}_{4}}$ - gas phase}

\begin{tabular}{|c|c|c|c|c|c|}
\hline \multirow{2}{*}{$\begin{array}{l}\text { Center } \\
\text { Number }\end{array}$} & \multirow{2}{*}{\multicolumn{2}{|c|}{$\begin{array}{l}\text { Atomic } \\
\text { Number }\end{array}$}} & \multirow{2}{*}{$\begin{array}{l}\text { Atomic } \\
\text { Type }\end{array}$} & \multicolumn{2}{|c|}{ Coordinates (Angstroms } \\
\hline & & & & $\mathrm{X} \quad \mathrm{Y}$ & $\mathrm{Z}$ \\
\hline 1 & 46 & 0 & -0.140847 & -0.011702 & -0.176947 \\
\hline 2 & 7 & 0 & -0.137089 & 0.036324 & 1.938580 \\
\hline 3 & 6 & 0 & 1.090359 & 0.015512 & 2.494435 \\
\hline 4 & 6 & 0 & 2.179873 & 0.255528 & 1.610650 \\
\hline 5 & 7 & 0 & 1.917778 & 0.111155 & 0.296686 \\
\hline 6 & 1 & 0 & -0.901752 & -0.248404 & 2.561254 \\
\hline 7 & 1 & 0 & 1.253650 & -0.188083 & 3.552173 \\
\hline 8 & 1 & 0 & 3.158205 & 0.553742 & 1.985960 \\
\hline 9 & 1 & 0 & 2.677332 & 0.395352 & -0.332442 \\
\hline 10 & 6 & 0 & 0.062222 & 0.498707 & -2.335059 \\
\hline 11 & 6 & 0 & -0.220628 & 1.726459 & -1.717550 \\
\hline 12 & 1 & 0 & -0.715315 & -0.043976 & -2.868514 \\
\hline 13 & 1 & 0 & 1.082618 & 0.235807 & -2.607257 \\
\hline 14 & 1 & 0 & -1.232703 & 2.120343 & -1.680427 \\
\hline 15 & 1 & 0 & 0.572487 & 2.413797 & -1.435977 \\
\hline 16 & 6 & 0 & -1.509361 & -1.869810 & -0.452932 \\
\hline 17 & 6 & 0 & -2.251934 & -0.679964 & -0.413958 \\
\hline 18 & 1 & 0 & -1.370826 & -2.479307 & 0.435924 \\
\hline 19 & 1 & 0 & -1.219471 & -2.324241 & -1.396478 \\
\hline 20 & 1 & 0 & -2.763787 & -0.374086 & 0.496459 \\
\hline 21 & 1 & 0 & -2.629493 & -0.233611 & -1.331484 \\
\hline
\end{tabular}

$\begin{array}{lccc}\text { Maximum Force } & 0.000023 & 0.000450 & \text { YES } \\ \text { RMS Force } & 0.000006 & 0.000300 & \text { YES } \\ \text { Maximum Displacement } & 0.000879 & 0.001800 & \text { YES } \\ \text { RMS Displacement } & 0.000218 & 0.001200 & \text { YES }\end{array}$

Predicted change in Energy=-3.775446D-08

SCF Done: $\mathrm{E}(\mathrm{RB}+\mathrm{HF}-\mathrm{LYP})=-472.005352972$ A.U. after 2 cycles Convg $=0.5936 \mathrm{D}-08 \quad \mathrm{~S} * * 2=0.0000$

\section{$\underline{4-\mathrm{CH}_{2}} \underline{\mathrm{Cl}}_{2}$}




\begin{tabular}{ccccccc}
\multirow{2}{*}{$\begin{array}{c}\text { Center } \\
\text { Number }\end{array}$} & \multicolumn{2}{c}{ Atomic } & \multicolumn{2}{c}{ Atomic } & \multicolumn{2}{c}{ Coordinates (Angstroms) } \\
- & Number & Type & X & Y & $Z$ \\
\hline 1 & 46 & 0 & -0.248596 & 0.027662 & -0.328553 \\
2 & 7 & 0 & -0.241068 & -0.018035 & 1.869026 \\
3 & 6 & 0 & 0.927318 & -0.122920 & 2.444376 \\
4 & 6 & 0 & 2.097802 & -0.196733 & 1.552593 \\
5 & 7 & 0 & 1.859507 & -0.147625 & 0.268856 \\
6 & 1 & 0 & -1.050767 & 0.033634 & 2.499426 \\
7 & 1 & 0 & 1.074748 & -0.157467 & 3.525469 \\
8 & 1 & 0 & 3.095873 & -0.287515 & 1.985381 \\
9 & 1 & 0 & 2.682192 & -0.199828 & -0.344458 \\
10 & 6 & 0 & -2.026774 & 0.181059 & -1.488474 \\
11 & 6 & 0 & -0.885109 & 0.114040 & -2.357914 \\
12 & 1 & 0 & -2.665567 & -0.693674 & -1.358059 \\
13 & 1 & 0 & -2.530698 & 1.134343 & -1.322681 \\
14 & 1 & 0 & -0.656475 & -0.811539 & -2.888252 \\
15 & 1 & 0 & -0.522387 & 1.016516 & -2.852026
\end{tabular}

$\begin{array}{lccc}\text { Maximum Force } & 0.000009 & 0.000450 & \text { YES } \\ \text { RMS Force } & 0.000003 & 0.000300 & \text { YES } \\ \text { Maximum Displacement } & 0.001388 & 0.001800 & \text { YES } \\ \text { RMS Displacement } & 0.000291 & 0.001200 & \text { YES }\end{array}$

SCF Done: $\mathrm{E}(\mathrm{RB}+\mathrm{HF}-\mathrm{LYP})=-393.432190821$ A.U. after 10 cycles Convg $=0.9658 \mathrm{D}-08 \quad \mathrm{~S} * 2=0.0000$

\section{$\underline{\mathrm{C}}_{2} \underline{\mathrm{H}}_{4}-\mathrm{CH}_{2} \underline{\mathrm{Cl}_{2}}$}

\begin{tabular}{cccccc} 
Center & \multicolumn{2}{c}{ Atomic } & \multicolumn{2}{c}{ Atomic } & \multicolumn{3}{c}{ Coordinates (Angstron } \\
Number & Number & Type & X & Y & Z \\
-1 & 6 & 0 & 0.000000 & 0.000000 & -0.673953 \\
2 & 6 & 0 & 0.000000 & 0.000000 & 0.673953 \\
3 & 1 & 0 & 0.926500 & 0.000000 & 1.246501 \\
4 & 1 & 0 & 0.926500 & 0.000000 & -1.246501 \\
5 & 1 & 0 & -0.926500 & 0.000000 & 1.246501 \\
6 & 1 & 0 & -0.926500 & 0.000000 & -1.246501
\end{tabular}

$\begin{array}{lccc}\text { Maximum Force } & 0.000288 & 0.000450 & \text { YES } \\ \text { RMS Force } & 0.000173 & 0.000300 & \text { YES } \\ \text { Maximum Displacement } & 0.001373 & 0.001800 & \text { YES } \\ \text { RMS Displacement } & 0.000857 & 0.001200 & \text { YES } \\ \text { Predicted change in Energy=-7.915083D-07 } & \end{array}$


SCF Done: $\mathrm{E}(\mathrm{RB}+\mathrm{HF}-\mathrm{LYP})=-78.5787872133$ A.U. after 6 cycles

$$
\text { Convg }=0.9365 \mathrm{D}-08 \quad \mathrm{~S} * * 2=0.0000
$$

\section{$\left[4 / \mathrm{C}_{2} \underline{\mathrm{H}_{4}}\right]^{\ddagger}-\mathrm{CH}_{2} \underline{\mathrm{Cl}_{2}}$}

\begin{tabular}{|c|c|c|c|c|}
\hline \multirow{2}{*}{$\begin{array}{l}\text { Center } \\
\text { Number }\end{array}$} & \multirow{2}{*}{$\begin{array}{l}\text { Atomic } \\
\text { Numbe }\end{array}$} & \multirow{2}{*}{$\begin{array}{l}\text { Atomic } \\
\text { Type }\end{array}$} & \multicolumn{2}{|c|}{ Coordinates (Angstroms } \\
\hline & & & X $\quad Y$ & $\begin{array}{l}\mathrm{Z} \\
\mathrm{Z}\end{array}$ \\
\hline 1 & 6 & -2.750346 & 0.086497 & -0.793637 \\
\hline 2 & 6 & -2.706185 & 0.219480 & 0.552779 \\
\hline 3 & 46 & 0.127484 & 0.267860 & 0.184571 \\
\hline 4 & 6 & 0.388864 & 2.288764 & 0.861667 \\
\hline 5 & 6 & 0.206729 & 1.468243 & 2.012024 \\
\hline 6 & 7 & 0.101104 & -1.899316 & -0.028358 \\
\hline 7 & 6 & 0.898674 & -2.376770 & -0.968747 \\
\hline 8 & 6 & 1.744603 & -1.418679 & -1.652793 \\
\hline 9 & 7 & 1.419849 & -0.140436 & -1.511433 \\
\hline 10 & 1 & -2.693446 & -0.887060 & -1.275170 \\
\hline 11 & 1 & -2.838022 & 0.950365 & -1.449968 \\
\hline 12 & 1 & -2.644290 & -0.644143 & $3 \quad 1.211718$ \\
\hline 13 & 1 & -2.785192 & 1.193640 & 1.031460 \\
\hline 14 & 1 & -0.552038 & -2.581752 & 0.377819 \\
\hline 15 & 1 & 0.926801 & -3.429994 & -1.252729 \\
\hline 16 & 1 & 2.601698 & -1.760647 & -2.234734 \\
\hline 17 & 1 & 2.057782 & 0.520287 & -1.972609 \\
\hline 18 & 1 & -0.749710 & 1.450221 & 2.532059 \\
\hline 19 & 1 & 1.061379 & 1.159754 & 2.611913 \\
\hline 20 & 1 & -0.431720 & 2.912761 & 0.504050 \\
\hline 21 & 1 & 1.384341 & 2.643031 & 0.594638 \\
\hline
\end{tabular}

$\begin{array}{llll}\text { Maximum Force } & 0.000423 & 0.000450 & \text { YES } \\ \text { RMS Force } & 0.000079 & 0.000300 & \text { YES } \\ \text { Maximum Displacement } & 0.229275 & 0.001800 & \text { NO } \\ \text { RMS Displacement } & 0.066086 & 0.001200 & \text { NO } \\ \text { Predicted change in Energy }=6.757845 \mathrm{D}-07 & \end{array}$

SCF Done: $\mathrm{E}(\mathrm{RB}+\mathrm{HF}-\mathrm{LYP})=-472.003882501 \quad$ A.U. after 7 cycles Convg $=0.6363 \mathrm{D}-08 \quad \mathrm{~S} * 2=0.0000$

\section{$\underline{4 \cdot \mathrm{C}_{2}} \underline{\mathrm{H}}_{4}-\mathrm{CH}_{2} \underline{\mathrm{Cl}_{2}}$}

Center Atomic Atomic Coordinates (Angstroms) 


\begin{tabular}{cccccc} 
Number & Number & Type & $X$ & $Y$ & $Z$ \\
\hline 1 & 46 & 0 & -0.138170 & -0.011237 & -0.173202 \\
2 & 7 & 0 & -0.137949 & -0.011906 & 1.933876 \\
3 & 6 & 0 & 1.092771 & -0.012578 & 2.487475 \\
4 & 6 & 0 & 2.171111 & 0.282508 & 1.608908 \\
5 & 7 & 0 & 1.910200 & 0.159296 & 0.290638 \\
6 & 1 & 0 & -0.891899 & -0.345754 & 2.547252 \\
7 & 1 & 0 & 1.259232 & -0.241528 & 3.539021 \\
8 & 1 & 0 & 3.141414 & 0.604676 & 1.983887 \\
9 & 1 & 0 & 2.654922 & 0.492603 & -0.334201 \\
10 & 6 & 0 & 0.069786 & 0.468003 & -2.328817 \\
11 & 6 & 0 & -0.210005 & 1.708305 & -1.734823 \\
12 & 1 & 0 & -0.708035 & -0.080029 & -2.856739 \\
13 & 1 & 0 & 1.090594 & 0.201590 & -2.596085 \\
14 & 1 & 0 & -1.220940 & 2.106814 & -1.709965 \\
15 & 1 & 0 & 0.586552 & 2.398997 & -1.471198 \\
16 & 6 & 0 & -1.528445 & -1.852749 & -0.448100 \\
17 & 6 & 0 & -2.249355 & -0.649363 & -0.407042 \\
18 & 1 & 0 & -1.407150 & -2.467316 & 0.439957 \\
19 & 1 & 0 & -1.250474 & -2.311903 & -1.393263 \\
20 & 1 & 0 & -2.756792 & -0.340875 & 0.504999 \\
21 & 1 & 0 & -2.622547 & -0.196834 & -1.323555 \\
----------------------------------------------------------
\end{tabular}

$\begin{array}{lccc}\text { Maximum Force } & 0.000068 & 0.000450 & \text { YES } \\ \text { RMS Force } & 0.000021 & 0.000300 & \text { YES } \\ \text { Maximum Displacement } & 0.001413 & 0.001800 & \text { YES } \\ \text { RMS Displacement } & 0.000370 & 0.001200 & \text { YES }\end{array}$

Predicted change in Energy=-2.567640D-06

SCF Done: $\mathrm{E}(\mathrm{RB}+\mathrm{HF}-\mathrm{LYP})=-472.012967727$ A.U. after 7 cycles Convg $=0.5904 \mathrm{D}-08 \quad \mathrm{~S} * * 2=0.0000$

\section{4-toluene}

\begin{tabular}{|c|c|c|c|c|}
\hline \multirow{2}{*}{$\begin{array}{l}\text { Center } \\
\text { Number }\end{array}$} & \multirow{2}{*}{$\begin{array}{l}\text { Atomic } \\
\text { Number }\end{array}$} & \multirow{2}{*}{$\begin{array}{r}\text { Atomic } \\
\text { Type }\end{array}$} & \multicolumn{2}{|c|}{ Coordinates (Angstroms) } \\
\hline & & & $\mathrm{X} \quad \mathrm{Y}$ & $\mathrm{Z}$ \\
\hline 1 & 46 & -0.249205 & 0.028046 & -0.327570 \\
\hline 2 & 7 & -0.237912 & -0.018914 & 1.867599 \\
\hline 3 & 6 & 0.930745 & -0.125117 & 2.444551 \\
\hline 4 & 6 & 2.098405 & -0.195432 & 1.552849 \\
\hline 5 & 7 & 1.854901 & -0.147168 & 0.268862 \\
\hline 6 & 1 & -1.047465 & 0.030020 & 2.493747 \\
\hline
\end{tabular}




$\begin{array}{cccccc}7 & 1 & 0 & 1.079004 & -0.163095 & 3.525079 \\ 8 & 1 & 0 & 3.097179 & -0.282332 & 1.983895 \\ 9 & 1 & 0 & 2.672237 & -0.195717 & -0.347166 \\ 10 & 6 & 0 & -2.026605 & 0.183003 & -1.492784 \\ 11 & 6 & 0 & -0.884935 & 0.111147 & -2.358673 \\ 12 & 1 & 0 & -2.665308 & -0.690030 & -1.358110 \\ 13 & 1 & 0 & -2.526156 & 1.137371 & -1.326043 \\ 14 & 1 & 0 & -0.654716 & -0.816355 & -2.883070 \\ 15 & 1 & 0 & -0.515948 & 1.011006 & -2.850999\end{array}$

$\begin{array}{lccc}\text { Maximum Force } & 0.000190 & 0.000450 & \text { YES } \\ \text { RMS Force } & 0.000041 & 0.000300 & \text { YES } \\ \text { Maximum Displacement } & 0.000151 & 0.001800 & \text { YES } \\ \text { RMS Displacement } & 0.000042 & 0.001200 & \text { YES }\end{array}$

Predicted change in Energy=-1.162617D-06

SCF Done: $\mathrm{E}(\mathrm{RB}+\mathrm{HF}-\mathrm{LYP})=-393.427141536$ A.U. after 12 cycles Convg $=0.9947 \mathrm{D}-08 \quad \mathrm{~S} * * 2=0.0000$

\section{$\underline{\mathbf{C}}_{2} \underline{H}_{4}$-toluene}

\begin{tabular}{|c|c|c|c|}
\hline Center & Atomic & Atomic & Coordinates (Angstroms) \\
\hline Number & Number & Type & $\begin{array}{lll}X & Y & Z\end{array}$ \\
\hline 1 & 6 & 0.000000 & -0.673785 \\
\hline 2 & 6 & 0.000000 & 0.673785 \\
\hline 3 & 1 & 0.926107 & 0.000000 \\
\hline 4 & 1 & 0.926107 & $0.000000 \quad-1.246313$ \\
\hline 5 & 1 & -0.926107 & $0.000000 \quad 1.246313$ \\
\hline 6 & 1 & -0.926107 & $0.000000-1.246313$ \\
\hline
\end{tabular}

$\begin{array}{llll}\text { Maximum Force } & 0.000290 & 0.000450 & \text { YES } \\ \text { RMS Force } & 0.000171 & 0.000300 & \text { YES } \\ \text { Maximum Displacement } & 0.001344 & 0.001800 & \text { YES } \\ \text { RMS Displacement } & 0.000831 & 0.001200 & \text { YES }\end{array}$

Predicted change in Energy=-7.655922D-07

SCF Done: $\mathrm{E}(\mathrm{RB}+\mathrm{HF}-\mathrm{LYP})=-78.5785191586$ A.U. after 7 cycles Convg $=0.1050 \mathrm{D}-08 \quad \mathrm{~S} * * 2=0.0000$

\section{$\left[4 / \mathbf{C}_{2}{\underline{\mathbf{H}_{4}}}_{4}\right]^{*}$ - toluene}




\begin{tabular}{cccccc} 
Center & \multicolumn{2}{c}{ Atomic } & \multicolumn{2}{c}{ Atomic } & \multicolumn{3}{c}{ Coordinates (Angstroms) } \\
Number & Number & Type & X & Y & Z \\
---------------- \\
1 & 6 & 0 & 0.000000 & 0.000000 & 0.000000 \\
2 & 6 & 0 & 0.000000 & 0.000000 & 1.352768 \\
3 & 46 & 0 & 2.893676 & 0.000000 & 0.272616 \\
4 & 7 & 0 & 3.705439 & -0.844061 & -1.536728 \\
5 & 6 & 0 & 4.140822 & -2.093217 & -1.411854 \\
6 & 6 & 0 & 3.640532 & -2.832329 & -0.273239 \\
7 & 7 & 0 & 3.063995 & -2.124844 & 0.687730 \\
8 & 6 & 0 & 3.161057 & 2.135641 & 0.246773 \\
9 & 6 & 0 & 3.333153 & 1.691914 & 1.587996 \\
10 & 1 & 0 & 0.019580 & -0.923359 & -0.574008 \\
11 & 1 & 0 & -0.048239 & 0.924269 & -0.571060 \\
12 & 1 & 0 & 0.028059 & -0.925260 & 1.924728 \\
13 & 1 & 0 & -0.043141 & 0.923368 & 1.925972 \\
14 & 1 & 0 & 2.634529 & -2.677248 & 1.437363 \\
15 & 1 & 0 & 3.734751 & -3.918647 & -0.242939 \\
16 & 1 & 0 & 4.846262 & -2.557166 & -2.101903 \\
17 & 1 & 0 & 4.104169 & -0.320470 & -2.322932 \\
18 & 1 & 0 & 2.535642 & 1.816346 & 2.317907 \\
19 & 1 & 0 & 4.329389 & 1.566802 & 2.006777 \\
20 & 1 & 0 & 2.236690 & 2.628936 & -0.052332 \\
21 & 1 & 0 & 4.030206 & 2.385644 & -0.360254
\end{tabular}

$\begin{array}{llll}\text { Maximum Force } & 0.000436 & 0.000450 & \text { YES } \\ \text { RMS Force } & 0.000099 & 0.000300 & \text { YES } \\ \text { Maximum Displacement } & 0.013746 & 0.001800 & \text { NO } \\ \text { RMS Displacement } & 0.004864 & 0.001200 & \text { NO }\end{array}$

Predicted change in Energy= 6.607469D-07

SCF Done: $\mathrm{E}(\mathrm{RB}+\mathrm{HF}-\mathrm{LYP})=-472.000118089$ A.U. after 7 cycles Convg $=0.5730 \mathrm{D}-08 \quad \mathrm{~S} * * 2=0.0000$

\section{$\underline{4 \cdot \mathrm{C}_{2}} \underline{\mathrm{H}}_{4}$ - toluene}

\begin{tabular}{cccccc} 
Center & \multicolumn{2}{c}{ Atomic } & \multicolumn{2}{c}{ Atomic } & \multicolumn{2}{c}{ Coordinates } \\
Number & Number & Type & X & Y & Z \\
--------- & \\
1 & 46 & 0 & -0.137861 & -0.011897 & -0.173197 \\
2 & 7 & 0 & -0.138609 & -0.011125 & 1.931609 \\
3 & 6 & 0 & 1.091504 & -0.008104 & 2.486666 \\
4 & 6 & 0 & 2.169507 & 0.283295 & 1.608214 \\
5 & 7 & 0 & 1.908199 & 0.156228 & 0.290335
\end{tabular}




$\begin{array}{cccccc}6 & 1 & 0 & -0.892486 & -0.342296 & 2.542645 \\ 7 & 1 & 0 & 1.258459 & -0.232903 & 3.537711 \\ 8 & 1 & 0 & 3.138799 & 0.604280 & 1.983166 \\ 9 & 1 & 0 & 2.651749 & 0.482491 & -0.335552 \\ 10 & 6 & 0 & 0.068089 & 0.467998 & -2.327475 \\ 11 & 6 & 0 & -0.209714 & 1.706746 & -1.729780 \\ 12 & 1 & 0 & -0.710951 & -0.077545 & -2.855040 \\ 13 & 1 & 0 & 1.086897 & 0.199136 & -2.597229 \\ 14 & 1 & 0 & -1.219780 & 2.105254 & -1.700334 \\ 15 & 1 & 0 & 0.586150 & 2.395720 & -1.462340 \\ 16 & 6 & 0 & -1.525294 & -1.850606 & -0.449558 \\ 17 & 6 & 0 & -2.247914 & -0.648419 & -0.408225 \\ 18 & 1 & 0 & -1.400371 & -2.465107 & 0.437173 \\ 19 & 1 & 0 & -1.243927 & -2.307754 & -1.393854 \\ 20 & 1 & 0 & -2.756458 & -0.339352 & 0.502268 \\ 21 & 1 & 0 & -2.620644 & -0.195848 & -1.324192 \\ ------------------------------------------------------------------\end{array}$

$\begin{array}{llll}\text { Maximum Force } & 0.000186 & 0.000450 & \text { YES } \\ \text { RMS Force } & 0.000028 & 0.000300 & \text { YES } \\ \text { Maximum Displacement } & 0.000688 & 0.001800 & \text { YES } \\ \text { RMS Displacement } & 0.000183 & 0.001200 & \text { YES } \\ \text { Predicted change in Energy=-2.967405D-06 } & \end{array}$

SCF Done: $\mathrm{E}(\mathrm{RB}+\mathrm{HF}-\mathrm{LYP})=-472.009046346 \quad$ A.U. after 7 cycles Convg $=0.5862 \mathrm{D}-08 \quad \mathrm{~S} * * 2=0.0000$ 
Gaussian98 Results (xyz cooridinates, optimization gradients, total energy) for the SelfExchange of $\mathrm{C}_{2} \mathrm{H}_{3} \mathrm{NO}_{2}$ with 5 using a LANL2DZ basis set (Basis $A$ ).

\section{5 -gas phase}

\begin{tabular}{|c|c|c|c|c|c|}
\hline \multirow{2}{*}{$\begin{array}{l}\text { Center } \\
\text { Number }\end{array}$} & \multirow{2}{*}{$\begin{array}{l}\text { Atomic } \\
\text { Number }\end{array}$} & \multirow{2}{*}{\multicolumn{2}{|c|}{$\begin{array}{l}\text { Atomic } \\
\text { Type }\end{array}$}} & \multicolumn{2}{|c|}{ Coordinates (Angstroms) } \\
\hline & & & & X $\quad Y$ & Z Z \\
\hline 1 & 6 & 0 & 1.300725 & -1.671023 & -0.248817 \\
\hline 2 & 6 & 0 & 1.773221 & -0.384182 & -0.673813 \\
\hline 3 & 46 & 0 & -0.302586 & -0.300856 & -0.188948 \\
\hline 4 & 7 & 0 & -1.528204 & 1.508605 & -0.234643 \\
\hline 5 & 6 & 0 & -2.779727 & 1.364601 & 0.104900 \\
\hline 6 & 6 & 0 & -3.208260 & -0.006702 & 0.443745 \\
\hline 7 & 7 & 0 & -2.289160 & -0.932587 & 0.387042 \\
\hline 8 & 1 & 0 & -2.578851 & -1.886451 & 0.623333 \\
\hline 9 & 1 & 0 & -4.246158 & -0.192927 & 0.724375 \\
\hline 10 & 1 & 0 & -3.502229 & 2.180878 & 0.154041 \\
\hline 11 & 1 & 0 & -1.213357 & 2.456670 & -0.464543 \\
\hline 12 & 1 & 0 & 1.564286 & -2.023549 & 0.745911 \\
\hline 13 & 1 & 0 & 1.159353 & -2.439407 & -1.006989 \\
\hline 14 & 7 & 0 & 2.588190 & 0.446062 & 0.218871 \\
\hline 15 & 1 & 0 & 1.978374 & -0.130932 & -1.708546 \\
\hline 16 & 8 & 0 & 3.114867 & 1.504801 & -0.289628 \\
\hline 17 & 8 & 0 & 2.740881 & 0.108244 & 1.448257 \\
\hline
\end{tabular}

$\begin{array}{lccc}\text { Maximum Force } & 0.000029 & 0.000450 & \text { YES } \\ \text { RMS Force } & 0.000005 & 0.000300 & \text { YES } \\ \text { Maximum Displacement } & 0.001417 & 0.001800 & \text { YES } \\ \text { RMS Displacement } & 0.000339 & 0.001200 & \text { YES } \\ \text { Predicted change in Energy=-8.810047D-09 } & \end{array}$

SCF Done: $\mathrm{E}(\mathrm{RB}+\mathrm{HF}-\mathrm{LYP})=-597.907323289 \quad$ A.U. after 2 cycles Convg $=0.1320 \mathrm{D}-08 \quad \mathrm{~S} * * 2=0.0000$

\section{$\underline{\mathrm{C}}_{2} \underline{\mathrm{H}}_{3} \underline{\mathrm{NO}_{2}}=$ gas phase}

\begin{tabular}{lccccc} 
Center & \multicolumn{2}{c}{ Atomic } & \multicolumn{3}{c}{ Coordinates (Angstroms) } \\
Number & Number & X & Y & Z \\
\hline 1 & 6 & 0 & -1.899869 & 0.034183 & 0.000176 \\
2 & 6 & 0 & -0.748907 & -0.655792 & -0.000290
\end{tabular}




$\begin{array}{rrrrrr}3 & 7 & 0 & 0.552505 & 0.029445 & -0.000010 \\ 4 & 8 & 0 & 1.585232 & -0.728286 & 0.000254 \\ 5 & 8 & 0 & 0.591781 & 1.306716 & -0.000189 \\ 6 & 1 & 0 & -1.901193 & 1.120513 & 0.000879 \\ 7 & 1 & 0 & -2.849688 & -0.491126 & 0.000150 \\ 8 & 1 & 0 & -0.640099 & -1.733286 & -0.000793\end{array}$

$\begin{array}{llll}\text { Maximum Force } & 0.000163 & 0.000450 & \text { YES } \\ \text { RMS Force } & 0.000045 & 0.000300 & \text { YES } \\ \text { Maximum Displacement } & 0.001334 & 0.001800 & \text { YES } \\ \text { RMS Displacement } & 0.000479 & 0.001200 & \text { YES } \\ \text { Predicted change in Energy=-7.751138D-08 } & \end{array}$

Predicted change in Energy=-7.751138D-08

SCF Done: $\mathrm{E}(\mathrm{RB}+\mathrm{HF}-\mathrm{LYP})=-283.048870681 \quad$ A.U. after 1 cycles Convg $=0.7395 \mathrm{D}-08 \quad \mathrm{~S} * * 2=0.0000$

\section{$\left[5 / \mathrm{C}_{2} \underline{\mathrm{H}}_{3} \underline{\mathrm{NO}}_{2}\right]^{\ddagger}$ - gas phase}

\begin{tabular}{|c|c|c|c|c|c|}
\hline \multirow{2}{*}{$\begin{array}{l}\text { Center } \\
\text { Number }\end{array}$} & \multirow{2}{*}{\multicolumn{2}{|c|}{$\begin{array}{l}\text { Atomic } \\
\text { Number }\end{array}$}} & \multirow{2}{*}{$\begin{array}{l}\text { Atomic } \\
\text { Type }\end{array}$} & \multicolumn{2}{|c|}{ Coordinates (Angstrom } \\
\hline & & & & $\mathrm{X} \quad \mathrm{Y}$ & $\mathrm{Z}$ \\
\hline 1 & 46 & 0 & 0.069253 & 0.410362 & -0.128512 \\
\hline 2 & 6 & 0 & -0.368276 & 0.145038 & 2.422505 \\
\hline 3 & 6 & 0 & 0.932900 & 0.399323 & 2.013586 \\
\hline 4 & 1 & 0 & -1.114905 & 0.898572 & 2.635252 \\
\hline 5 & 1 & 0 & 1.317772 & 1.411155 & 2.095951 \\
\hline 6 & 1 & 0 & 1.655548 & -0.410683 & 1.979694 \\
\hline 7 & 7 & 0 & -0.845702 & -1.192830 & 2.594727 \\
\hline 8 & 8 & 0 & -2.130268 & -1.359440 & 2.679024 \\
\hline 9 & 8 & 0 & -0.015398 & -2.182633 & 2.608307 \\
\hline 10 & 6 & 0 & 0.805431 & 2.339654 & -0.659392 \\
\hline 11 & 6 & 0 & 1.794419 & 1.381730 & -1.022796 \\
\hline 12 & 1 & 0 & 0.175859 & 2.764842 & -1.436482 \\
\hline 13 & 1 & 0 & 0.980865 & 2.963815 & 0.214047 \\
\hline 14 & 1 & 0 & 2.714479 & 1.212077 & -0.475629 \\
\hline 15 & 7 & 0 & 1.951823 & 0.970373 & -2.423816 \\
\hline 16 & 8 & 0 & 1.082596 & 1.331193 & -3.296097 \\
\hline 17 & 8 & 0 & 2.982207 & 0.255105 & -2.701576 \\
\hline 18 & 7 & 0 & -2.181586 & 0.188918 & -0.442589 \\
\hline 19 & 6 & 0 & -2.605089 & -1.043123 & -0.437833 \\
\hline 20 & 6 & 0 & -1.592473 & -2.118738 & -0.340706 \\
\hline 21 & 7 & 0 & -0.340810 & -1.766868 & -0.274610 \\
\hline 22 & 1 & 0 & -2.903175 & 0.916012 & -0.494133 \\
\hline 23 & 1 & 0 & -3.658057 & -1.326548 & -0.485375 \\
\hline
\end{tabular}




$\begin{array}{rrrrrr}24 & 1 & 0 & -1.932644 & -3.154194 & -0.305596 \\ 25 & 1 & 0 & 0.337978 & -2.525946 & -0.161612\end{array}$

\begin{tabular}{llll}
\multicolumn{1}{c}{ Item Value } & Threshold & Converged? & \\
Maximum Force & 0.000029 & 0.000450 & YES \\
RMS Force & 0.000004 & 0.000300 & YES \\
Maximum Displacement & 0.001535 & 0.001800 & YES \\
RMS Displacement & 0.000301 & 0.001200 & YES \\
Predicted change in Energy $=-1.002826 \mathrm{D}-08$ &
\end{tabular}

SCF Done: $\mathrm{E}(\mathrm{RB}+\mathrm{HF}-\mathrm{LYP})=-880.961854972$ A.U. after 2 cycles Convg $=0.1448 \mathrm{D}-08 \quad \mathrm{~S} * * 2=0.0000$

\section{$\underline{5 \cdot \mathbf{C}_{2}} \underline{\underline{H_{3}}} \underline{\underline{N O}} \underline{\underline{2}} \underline{\text { Isomers- gas phase }}$}

\section{$5 \cdot \mathrm{C}_{2} \mathrm{H}_{3} \mathrm{NO}_{2}-\mathrm{A}$}

\begin{tabular}{|c|c|c|c|c|c|}
\hline \multirow{2}{*}{$\begin{array}{l}\text { Center } \\
\text { Number }\end{array}$} & \multirow{2}{*}{\multicolumn{2}{|c|}{$\begin{array}{l}\text { Atomic } \\
\text { Number }\end{array}$}} & \multicolumn{3}{|c|}{ Coordinates (Angstroms) } \\
\hline & & & $\mathrm{X}$ & $\mathrm{Y}$ & $\mathrm{Z}$ \\
\hline 1 & 6 & 0 & -2.275186 & -0.205777 & -0.511876 \\
\hline 2 & 6 & 0 & -2.282119 & -0.039049 & 0.895294 \\
\hline 3 & 46 & 0 & -0.175034 & -0.195226 & -0.072029 \\
\hline 4 & 6 & 0 & 0.026749 & -2.304781 & -0.408333 \\
\hline 5 & 6 & 0 & 0.660014 & -1.773783 & -1.559408 \\
\hline 6 & 7 & 0 & 1.808085 & 0.141701 & 0.822506 \\
\hline 7 & 6 & 0 & 2.204029 & 1.383536 & 0.849556 \\
\hline 8 & 6 & 0 & 1.187940 & 2.406874 & 0.538736 \\
\hline 9 & 7 & 0 & 0.098462 & 1.989006 & -0.042735 \\
\hline 10 & 1 & 0 & 2.516581 & -0.591807 & 0.943393 \\
\hline 11 & 1 & 0 & 3.228542 & 1.687852 & 1.068929 \\
\hline 12 & 1 & 0 & 1.367280 & 3.447662 & 0.812517 \\
\hline 13 & 1 & 0 & -0.660931 & 2.665644 & -0.184444 \\
\hline 14 & 1 & 0 & -2.544756 & -1.177747 & -0.916035 \\
\hline 15 & 1 & 0 & -2.500895 & 0.651169 & -1.140855 \\
\hline 16 & 1 & 0 & -2.405009 & -0.842069 & 1.611286 \\
\hline 17 & 7 & 0 & -2.513140 & 1.255432 & 1.491606 \\
\hline 18 & 7 & 0 & 2.099226 & -1.719119 & -1.660291 \\
\hline 19 & 1 & 0 & 0.181644 & -1.643311 & -2.522073 \\
\hline 20 & 1 & 0 & 0.644344 & -2.723116 & 0.381914 \\
\hline 21 & 1 & 0 & -0.963016 & -2.739098 & -0.520066 \\
\hline 22 & 8 & 0 & 2.602479 & -1.340593 & -2.781733 \\
\hline 23 & 8 & 0 & 2.829975 & -2.052579 & -0.640415 \\
\hline 24 & 8 & 0 & -2.628883 & 1.308082 & 2.771447 \\
\hline
\end{tabular}


$\begin{array}{llllll}25 & 8 & 0 & -2.602224 & 2.306836 & 0.735372\end{array}$

$\begin{array}{lccc}\text { Maximum Force } & 0.000022 & 0.000450 & \text { YES } \\ \text { RMS Force } & 0.000005 & 0.000300 & \text { YES } \\ \text { Maximum Displacement } & 0.001495 & 0.001800 & \text { YES } \\ \text { RMS Displacement } & 0.000240 & 0.001200 & \text { YES }\end{array}$

Predicted change in Energy=-1.920963D-08

SCF Done: $\mathrm{E}(\mathrm{RB}+\mathrm{HF}-\mathrm{LYP})=-880.974979875 \quad$ A.U. after 12 cycles Convg $=0.9754 \mathrm{D}-08 \quad \mathrm{~S} * * 2=0.0000$

\section{$5 \cdot \mathrm{C}_{2} \mathrm{H}_{3} \mathrm{NO}_{2}-\mathrm{B}$}

\begin{tabular}{|c|c|c|c|c|c|}
\hline \multirow{2}{*}{$\begin{array}{l}\text { Center } \\
\text { Number }\end{array}$} & \multirow{2}{*}{\multicolumn{2}{|c|}{$\begin{array}{l}\text { Atomic } \\
\text { Number }\end{array}$}} & \multicolumn{3}{|c|}{ Coordinates (Angstroms) } \\
\hline & & & X & Y & $\mathrm{Z}$ \\
\hline 1 & 6 & 0 & -0.050401 & -0.301995 & -2.214035 \\
\hline 2 & 46 & 0 & -0.034168 & -0.367122 & -0.058531 \\
\hline 3 & 7 & 0 & 2.115093 & -0.525662 & 0.232304 \\
\hline 4 & 6 & & 2.557825 & -0.153053 & 1.404699 \\
\hline 5 & 6 & 0 & 1.549484 & 0.057446 & 2.453934 \\
\hline 6 & 7 & 0 & 0.311996 & 0.173034 & 2.049278 \\
\hline 7 & 6 & 0 & 0.275080 & 1.049208 & -1.942952 \\
\hline 8 & 7 & & 1.630344 & 1.517264 & -2.045954 \\
\hline 9 & 8 & & 2.589527 & 0.672501 & -2.293448 \\
\hline 10 & 6 & 0 & -2.132158 & -0.954624 & 0.08172 \\
\hline 11 & 6 & 0 & -1.376795 & -2.098519 & 0.421791 \\
\hline 12 & 1 & & -1.246105 & -2.379521 & 1.461114 \\
\hline 13 & 8 & & 1.842944 & 2.777939 & -1.894281 \\
\hline 14 & 1 & & -0.432130 & 0.273694 & 2.751000 \\
\hline 15 & 1 & 0 & 1.855573 & 0.113966 & 3.499629 \\
\hline 16 & 1 & 0 & 3.611207 & 0.035746 & 1.616726 \\
\hline 17 & 1 & & 2.784683 & -0.561981 & -0.545975 \\
\hline 18 & 1 & & -1.060731 & -0.521877 & -2.549390 \\
\hline 19 & 1 & 0 & 0.730526 & -0.960139 & $-2.58587\}$ \\
\hline 20 & 1 & 0 & -0.440260 & 1.860462 & -1.895353 \\
\hline 21 & 1 & 0 & -1.208222 & -2.862297 & -0.330706 \\
\hline 22 & 7 & & -2.810337 & -0.170075 & 1.129209 \\
\hline 23 & 1 & & -2.638768 & -0.820946 & -0.866784 \\
\hline 24 & 8 & 0 & -3.696993 & 0.665086 & 0.743199 \\
\hline 25 & 8 & 0 & -2.491967 & -0.345802 & 2.363676 \\
\hline
\end{tabular}

Maximum Force $\quad 0.000014 \quad 0.000450 \quad$ YES 
$\begin{array}{lccc}\text { RMS Force } & 0.000004 & 0.000300 & \text { YES } \\ \text { Maximum Displacement } & 0.020681 & 0.001800 & \text { NO } \\ \text { RMS Displacement } & 0.004318 & 0.001200 & \text { NO }\end{array}$

Predicted change in Energy=-6.499932D-07

SCF Done: $\mathrm{E}(\mathrm{RB}+\mathrm{HF}-\mathrm{LYP})=-880.971230191 \quad$ A.U. after 15 cycles Convg $=0.8539 \mathrm{D}-08 \quad \mathrm{~S} * 2=0.0000$

\section{$5 \cdot \mathrm{C}_{2} \mathrm{H}_{3} \mathrm{NO}_{2}-\mathrm{C}$}

\begin{tabular}{|c|c|c|c|c|c|}
\hline \multirow{2}{*}{$\begin{array}{l}\text { Center } \\
\text { Number }\end{array}$} & \multirow{2}{*}{\multicolumn{2}{|c|}{$\begin{array}{l}\text { Atomic } \\
\text { Number }\end{array}$}} & \multicolumn{3}{|c|}{ Coordinates (Angstroms) } \\
\hline & & & $\mathrm{X}$ & $\mathrm{Y}$ & Z \\
\hline 1 & 6 & 0 & -2.339948 & 0.291141 & 0.106142 \\
\hline 2 & 6 & 0 & -2.076527 & 0.139754 & 1.482891 \\
\hline 3 & 46 & 0 & -0.152302 & 0.273885 & 0.332896 \\
\hline 4 & 6 & 0 & 0.227269 & -1.855280 & 0.017388 \\
\hline 5 & 6 & & 0.889060 & -1.335382 & -1.110661 \\
\hline 6 & 7 & 0 & 1.702757 & 0.671761 & 1.359334 \\
\hline 7 & 6 & 0 & 2.146771 & 1.902339 & 1.290084 \\
\hline 8 & 6 & 0 & 1.220381 & 2.914329 & 0.778554 \\
\hline 9 & 7 & & 0.152668 & 2.473568 & 0.151219 \\
\hline 10 & 1 & & 2.386512 & -0.058581 & 1.588956 \\
\hline 11 & 1 & 0 & 3.166889 & 2.186743 & 1.550054 \\
\hline 12 & 1 & 0 & 1.450587 & 3.972579 & 0.910616 \\
\hline 13 & 1 & & -0.489 & 3.194 & -0.198270 \\
\hline 14 & 7 & & -2.882495 & -0.818723 & -0.689019 \\
\hline 15 & 1 & & -2.596962 & 1.237069 & -0.358182 \\
\hline 16 & 1 & 0 & -2.166884 & -0.836461 & 1.947622 \\
\hline 17 & 1 & 0 & -2.102226 & 1.009196 & 2.131832 \\
\hline 18 & 7 & & 2.322645 & -1.172133 & -1.127423 \\
\hline 19 & 1 & & 0.453483 & -1.243566 & -2.097675 \\
\hline 20 & 1 & 0 & 0.822659 & -2.202887 & 0.857417 \\
\hline 21 & 1 & 0 & -0.750855 & -2.311029 & -0.109005 \\
\hline 22 & 8 & 0 & 2.863706 & -0.785341 & -2.229160 \\
\hline 23 & 8 & & 3.017755 & -1.425091 & -0.060128 \\
\hline 24 & 8 & & -3.290535 & -0.535038 & -1.870145 \\
\hline 25 & 8 & 0 & -2.920728 & -2.000635 & -0.188287 \\
\hline
\end{tabular}

\begin{tabular}{|c|c|c|c|}
\hline & & & \\
\hline & & & \\
\hline & \multirow{2}{*}{0.006002} & \multirow{2}{*}{0.001800} & \\
\hline $\begin{array}{l}\text { MS Force } \\
\text { Iaximum Displacement } \\
\text { MS Displacement }\end{array}$ & & & \\
\hline
\end{tabular}


SCF Done: $\mathrm{E}(\mathrm{RB}+\mathrm{HF}-\mathrm{LYP})=-880.965567077$ A.U. after 34 cycles Convg $=0.7196 \mathrm{D}-08 \quad-\mathrm{V} / \mathrm{T}=2.1068$ $\mathrm{S} * * 2=0.0000$

$5 \cdot \mathrm{C}_{2} \mathrm{H}_{3} \mathrm{NO}_{2}-\mathrm{D}$

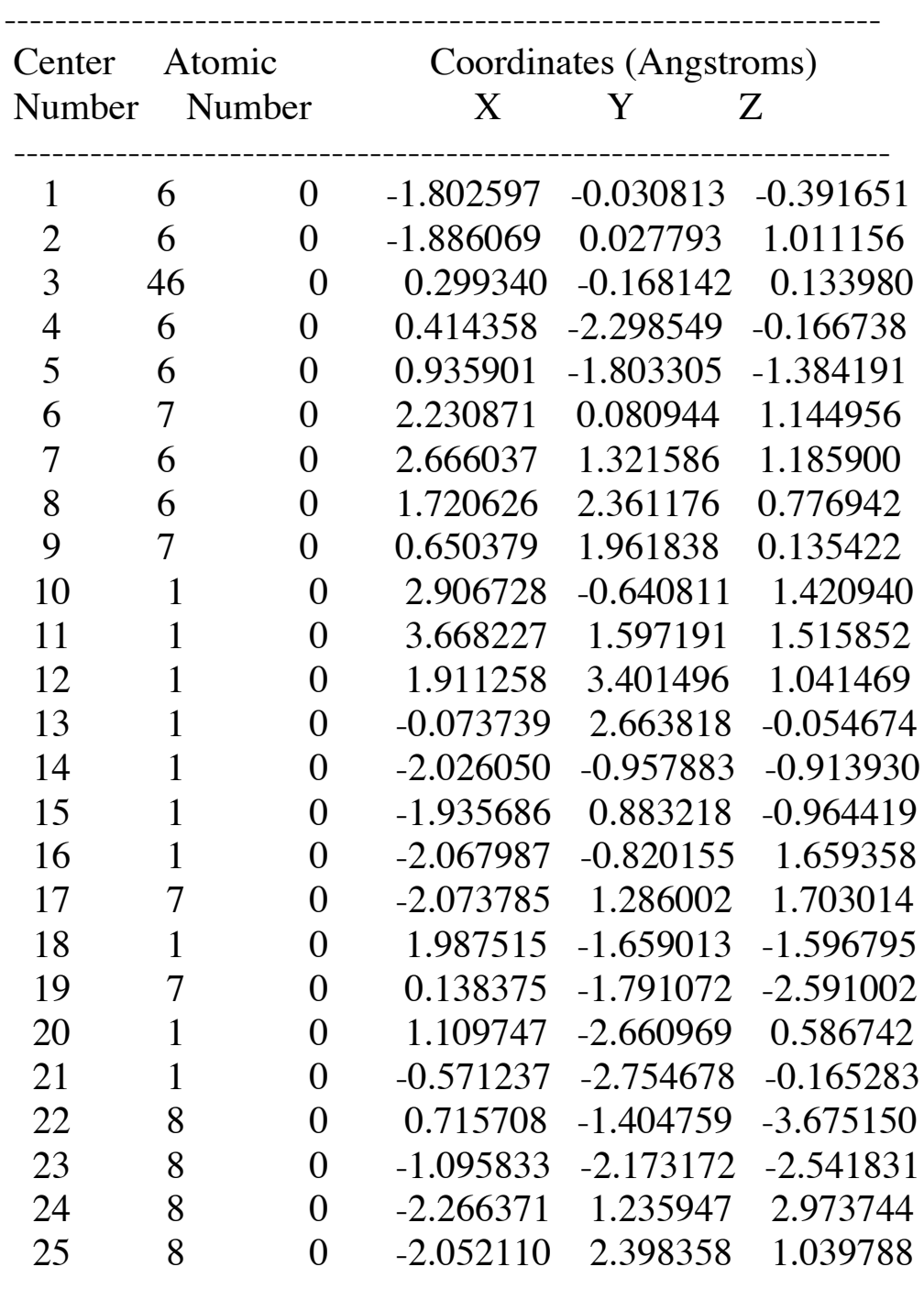

$\begin{array}{llll}\text { Maximum Force } & 0.000026 & 0.000450 & \text { YES } \\ \text { RMS Force } & 0.000008 & 0.000300 & \text { YES } \\ \text { Maximum Displacement } & 0.001792 & 0.001800 & \text { YES } \\ \text { RMS Displacement } & 0.000440 & 0.001200 & \text { YES } \\ \text { Predicted change in Energy=-1.374578D-08 } & \end{array}$

SCF Done: $\mathrm{E}(\mathrm{RB}+\mathrm{HF}-\mathrm{LYP})=-880.970845552$ A.U. after 12 cycles Convg $=0.6178 \mathrm{D}-08 \quad \mathrm{~S} * * 2=0.0000$ 


\section{$5 \cdot \mathrm{C}_{2} \mathrm{H}_{3} \mathrm{NO}_{2}-\mathrm{E}$}

\begin{tabular}{|c|c|c|c|c|c|}
\hline \multirow{2}{*}{$\begin{array}{l}\text { Center } \\
\text { Number }\end{array}$} & \multirow{2}{*}{\multicolumn{2}{|c|}{$\begin{array}{l}\text { Atomic } \\
\text { Number }\end{array}$}} & \multicolumn{3}{|c|}{ Coordinates (Angstroms) } \\
\hline & & & $\mathrm{X}$ & $\mathrm{Y} \quad \mathrm{Z}$ & $\mathrm{Z}$ \\
\hline 1 & 6 & 0 & 0.081182 & 0.225295 & -2.122689 \\
\hline 2 & 46 & 0 & 0.078152 & 0.014607 & 0.075333 \\
\hline 3 & 7 & 0 & 2.196405 & -0.256411 & 0.423460 \\
\hline 4 & 6 & 0 & 2.642199 & 0.209926 & 1.568432 \\
\hline 5 & 6 & 0 & 1.627232 & 0.591153 & 2.547141 \\
\hline 6 & 7 & 0 & 0.406378 & 0.744748 & 2.085534 \\
\hline 7 & 6 & 0 & 0.355119 & 1.513348 & -1.626921 \\
\hline 8 & 1 & 0 & 1.373654 & 1.883486 & -1.589107 \\
\hline 9 & 6 & 0 & -2.038719 & -0.592673 & 0.235803 \\
\hline 10 & 6 & 0 & -1.301197 & -1.690879 & 0.715283 \\
\hline 11 & 1 & 0 & -1.174941 & -1.846044 & 1.781034 \\
\hline 12 & 1 & 0 & -0.353821 & 0.933468 & 2.750980 \\
\hline 13 & 1 & 0 & 1.894706 & 0.719328 & 3.596719 \\
\hline 14 & 1 & 0 & 3.701601 & 0.330763 & 1.797889 \\
\hline 15 & 1 & 0 & 2.871777 & -0.461010 & -0.323767 \\
\hline 16 & 1 & 0 & -0.875651 & -0.073415 & -2.534370 \\
\hline 17 & 7 & 0 & 1.156251 & -0.618518 & -2.667390 \\
\hline 18 & 1 & 0 & -0.451908 & 2.235609 & -1.560357 \\
\hline 19 & 1 & 0 & -1.112512 & -2.529271 & 0.052798 \\
\hline 20 & 7 & 0 & -2.715002 & 0.326149 & 1.164724 \\
\hline 21 & 1 & 0 & -2.517111 & -0.563420 & -0.736064 \\
\hline 22 & 8 & 0 & 0.802105 & -1.651756 & -3.333486 \\
\hline 23 & 8 & 0 & 2.386546 & -0.302636 & -2.453959 \\
\hline 24 & 8 & 0 & -3.572387 & 1.132069 & 0.662658 \\
\hline 25 & 8 & 0 & -2.423003 & 0.296048 & 2.418831 \\
\hline
\end{tabular}

$\begin{array}{lccc}\text { Maximum Force } & 0.000011 & 0.000450 & \text { YES } \\ \text { RMS Force } & 0.000003 & 0.000300 & \text { YES } \\ \text { Maximum Displacement } & 0.001219 & 0.001800 & \text { YES } \\ \text { RMS Displacement } & 0.000313 & 0.001200 & \text { YES } \\ \text { Predicted change in Energy }=-1.095705 D-08 & \end{array}$

SCF Done: $\mathrm{E}(\mathrm{RB}+\mathrm{HF}-\mathrm{LYP})=-880.967783767$ A.U. after 12 cycles Convg $=0.8846 \mathrm{D}-08 \quad \mathrm{~S} * * 2=0.0000$

\section{$5 \cdot \mathrm{C}_{2} \mathrm{H}_{3} \mathrm{NO}_{2}-\mathrm{F}$}

Center Atomic Coordinates (Angstroms) 


\begin{tabular}{|c|c|c|c|c|c|}
\hline Number & & & $X$ & $\mathrm{Y}$ & \\
\hline 1 & 6 & 0 & 0.227173 & 0.176443 & -1.856125 \\
\hline 2 & 46 & 0 & 0.059872 & 0.050395 & 0.490901 \\
\hline 3 & 7 & 0 & 2.171358 & -0.220253 & 0.937109 \\
\hline 4 & 6 & 0 & 2.607744 & 0.464846 & 1.972584 \\
\hline 5 & 6 & 0 & 1.600241 & 1.127590 & 2.786883 \\
\hline 6 & 7 & 0 & 0.387647 & 1.208869 & 2.267588 \\
\hline 7 & 6 & 0 & 0.395000 & 1.470626 & -1.392529 \\
\hline 8 & 1 & 0 & 1.384708 & 1.891038 & -1.249123 \\
\hline 9 & 6 & 0 & -2.031217 & -0.628415 & 0.670732 \\
\hline 10 & 6 & 0 & -1.259422 & -1.762466 & 0.347058 \\
\hline 11 & 1 & 0 & -0.946226 & -2.428670 & 1.145077 \\
\hline 12 & 1 & 0 & -0.323275 & 1.671015 & 2.843966 \\
\hline 13 & 1 & 0 & 1.863376 & 1.542962 & 3.759952 \\
\hline 14 & 1 & 0 & 3.664949 & 0.585542 & 2.211732 \\
\hline 15 & 1 & 0 & 2.873670 & -0.588710 & 0.284019 \\
\hline 16 & 1 & 0 & -0.707421 & -0.222884 & -2.23204 \\
\hline 17 & 7 & 0 & 1.371956 & -0.667236 & -2.201337 \\
\hline 18 & 1 & 0 & -0.459513 & 2.138922 & -1.376570 \\
\hline 19 & 1 & 0 & -1.256430 & -2.161767 & -0.66174 \\
\hline 20 & 1 & 0 & -2.360454 & -0.394614 & 1.677109 \\
\hline 21 & 7 & 0 & -2.906199 & 0.013531 & -0.31390 \\
\hline 22 & 8 & 0 & -2.827557 & -0.317703 & -1.55391 \\
\hline 23 & 8 & 0 & -3.718213 & 0.903305 & 0.127915 \\
\hline 24 & 8 & 0 & 1.114675 & -1.784229 & -2.77208 \\
\hline 25 & 8 & 0 & 2.568853 & -0.274765 & -1.924627 \\
\hline
\end{tabular}

$\begin{array}{lccc}\text { Maximum Force } & 0.000002 & 0.000450 & \text { YES } \\ \text { RMS Force } & 0.000000 & 0.000300 & \text { YES } \\ \text { Maximum Displacement } & 0.009007 & 0.001800 & \text { NO } \\ \text { RMS Displacement } & 0.002134 & 0.001200 & \text { NO }\end{array}$

Predicted change in Energy=-9.153140D-07

SCF Done: $\mathrm{E}(\mathrm{RB}+\mathrm{HF}-\mathrm{LYP})=-880.963309612$ A.U. after 17 cycles Convg $=0.6958 \mathrm{D}-08 \quad \mathrm{~S} * * 2=0.0000$

\section{$5 \cdot \mathrm{C}_{2} \mathrm{H}_{3} \mathrm{NO}_{2}-\mathrm{G}$}

\begin{tabular}{|c|c|c|c|c|}
\hline Center & Atomic & \multicolumn{3}{|c|}{ Coordinates (Angstroms) } \\
\hline Number & Number & X & $\mathrm{Y} \quad \mathrm{Z}$ & \\
\hline 1 & 0 & -1.832418 & -0.0 & \\
\hline 2 & 6 & -1.752950 & -0.039759 & 1.512851 \\
\hline
\end{tabular}




$\begin{array}{cccccc}3 & 46 & 0 & 0.349756 & -0.086695 & 0.556357 \\ 4 & 6 & 0 & 0.493458 & -2.235420 & 0.364543 \\ 5 & 6 & 0 & 0.940973 & -1.860351 & -0.921280 \\ 6 & 7 & 0 & 2.162086 & 0.163806 & 1.670823 \\ 7 & 6 & 0 & 2.658977 & 1.390833 & 1.666044 \\ 8 & 6 & 0 & 1.796762 & 2.430216 & 1.132004 \\ 9 & 7 & 0 & 0.749361 & 2.021649 & 0.445458 \\ 10 & 1 & 0 & 2.787171 & -0.572489 & 2.016745 \\ 11 & 1 & 0 & 3.653396 & 1.631874 & 2.041096 \\ 12 & 1 & 0 & 2.013513 & 3.479966 & 1.328847 \\ 13 & 1 & 0 & 0.067741 & 2.728551 & 0.141683 \\ 14 & 1 & 0 & -2.044330 & -0.867276 & -0.524718 \\ 15 & 7 & 0 & -2.149294 & 1.255463 & -0.573043 \\ 16 & 1 & 0 & -1.843590 & -0.986617 & 2.034081 \\ 17 & 1 & 0 & -1.858212 & 0.873314 & 2.089373 \\ 18 & 1 & 0 & 1.975089 & -1.712718 & -1.204667 \\ 19 & 7 & 0 & 0.068952 & -1.959007 & -2.063813 \\ 20 & 1 & 0 & 1.234516 & -2.529251 & 1.104641 \\ 21 & 1 & 0 & -0.482513 & -2.703485 & 0.455606 \\ 22 & 8 & 0 & 0.577742 & -1.720550 & -3.222085 \\ 23 & 8 & 0 & -1.169448 & -2.299137 & -1.887957 \\ 24 & 8 & 0 & -2.513818 & 1.180341 & -1.790818 \\ 25 & 8 & 0 & -2.049236 & 2.369596 & 0.069479 \\ ----------------------------------------------------------\end{array}$

$\begin{array}{lccc}\text { Maximum Force } & 0.000117 & 0.000450 & \text { YES } \\ \text { RMS Force } & 0.000015 & 0.000300 & \text { YES } \\ \text { Maximum Displacement } & 0.001516 & 0.001800 & \text { YES } \\ \text { RMS Displacement } & 0.000235 & 0.001200 & \text { YES }\end{array}$

Predicted change in Energy=-6.627312D-08

SCF Done: $\mathrm{E}(\mathrm{RB}+\mathrm{HF}-\mathrm{LYP})=-880.965286267$ A.U. after 14 cycles Convg $=0.7346 \mathrm{D}-08 \quad \mathrm{~S} * * 2=0.0000$

\section{$5 \cdot \mathrm{C}_{2} \mathrm{H}_{3} \mathrm{NO}_{2}-\mathrm{H}$}

\begin{tabular}{|c|c|c|c|c|c|}
\hline \multirow{2}{*}{$\begin{array}{l}\text { Center } \\
\text { Number }\end{array}$} & \multirow{2}{*}{\multicolumn{2}{|c|}{$\begin{array}{l}\text { Atomic } \\
\text { Number }\end{array}$}} & \multicolumn{3}{|c|}{ Coordinates (Angstroms) } \\
\hline & & & $\mathrm{X}$ & $\mathrm{Y}$ & $\mathrm{Z}$ \\
\hline 1 & 6 & 0 & 0.759871 & 0.768005 & -1.871800 \\
\hline 2 & 46 & 0 & 0.318493 & 0.049141 & 0.309384 \\
\hline 3 & 7 & 0 & 1.676660 & -1.039290 & 1.614754 \\
\hline 4 & 6 & 0 & 2.396720 & -0.287115 & 2.423398 \\
\hline 5 & 6 & 0 & 2.347137 & 1.151567 & 2.199535 \\
\hline 6 & 7 & 0 & 1.407918 & 1.593205 & 1.386600 \\
\hline
\end{tabular}




$\begin{array}{cccccc}7 & 6 & 0 & -0.414332 & 1.301086 & -1.308798 \\ 8 & 1 & 0 & -0.385320 & 2.314980 & -0.916146 \\ 9 & 6 & 0 & -1.687703 & -0.933698 & 0.965006 \\ 10 & 6 & 0 & -1.062528 & -1.579432 & -0.115731 \\ 11 & 1 & 0 & -0.498351 & -2.488546 & 0.080554 \\ 12 & 1 & 0 & 1.404536 & 2.604978 & 1.213996 \\ 13 & 1 & 0 & 3.070024 & 1.801577 & 2.693117 \\ 14 & 1 & 0 & 3.006542 & -0.688109 & 3.233372 \\ 15 & 1 & 0 & 1.697371 & -2.047695 & 1.804715 \\ 16 & 7 & 0 & 0.718355 & -0.332504 & -2.816808 \\ 17 & 1 & 0 & 1.724541 & 1.260185 & -1.878705 \\ 18 & 1 & 0 & -1.390520 & 0.933011 & -1.612318 \\ 19 & 1 & 0 & -1.432172 & -1.456480 & -1.130084 \\ 20 & 1 & 0 & -1.590212 & -1.225847 & 2.003248 \\ 21 & 7 & 0 & -2.810764 & -0.031052 & 0.776461 \\ 22 & 8 & 0 & 1.828497 & -0.667654 & -3.377386 \\ 23 & 8 & 0 & -0.404720 & -0.909130 & -3.077183 \\ 24 & 8 & 0 & -3.233184 & 0.228894 & -0.412006 \\ 25 & 8 & 0 & -3.345251 & 0.457450 & 1.841557 \\ -------------------------------------------------------------------\end{array}$

$\begin{array}{lccc}\text { Maximum Force } & 0.000320 & 0.000450 & \text { YES } \\ \text { RMS Force } & 0.000092 & 0.000300 & \text { YES } \\ \text { Maximum Displacement } & 0.001506 & 0.001800 & \text { YES } \\ \text { RMS Displacement } & 0.000351 & 0.001200 & \text { YES } \\ \text { Predicted change in Energy=-1.277291D-06 } & \end{array}$

SCF Done: $\mathrm{E}(\mathrm{RB}+\mathrm{HF}-\mathrm{LYP})=-880.965761424$ A.U. after 19 cycles Convg $=0.7425 \mathrm{D}-08 \quad \mathrm{~S} * * 2=0.0000$

\section{$5 \cdot \mathrm{C}_{2} \mathrm{H}_{3} \mathrm{NO}_{2}-\mathrm{I}$}

\begin{tabular}{|c|c|c|c|c|c|}
\hline \multirow{2}{*}{$\begin{array}{l}\text { Center } \\
\text { Number }\end{array}$} & \multirow{2}{*}{\multicolumn{2}{|c|}{$\begin{array}{l}\text { Atomic } \\
\text { Number }\end{array}$}} & \multicolumn{3}{|c|}{ Coordinates (Angstroms) } \\
\hline & & & $\mathrm{X}$ & $\mathrm{Y}$ & $\mathrm{Z}$ \\
\hline 1 & 6 & 0 & -1.965996 & 0.331172 & 0.431807 \\
\hline 2 & 6 & 0 & -1.611017 & 0.191366 & 1.786474 \\
\hline 3 & 46 & 0 & 0.251611 & 0.245632 & 0.542318 \\
\hline 4 & 6 & 0 & 0.538920 & -1.891365 & 0.126315 \\
\hline 5 & 6 & 0 & 1.275169 & -1.278396 & -0.901400 \\
\hline 6 & 7 & 0 & 2.076115 & 0.641920 & 1.704796 \\
\hline 7 & 6 & 0 & 2.560311 & 1.860806 & 1.587841 \\
\hline 8 & 6 & 0 & 1.735403 & 2.840040 & 0.888101 \\
\hline 9 & 7 & 0 & 0.689419 & 2.379381 & 0.235547 \\
\hline 10 & 1 & 0 & 2.690165 & -0.040917 & 2.163869 \\
\hline
\end{tabular}




$\begin{array}{rrrrrr}11 & 1 & 0 & 3.537735 & 2.159575 & 1.969384 \\ 12 & 1 & 0 & 2.013710 & 3.894427 & 0.917252 \\ 13 & 1 & 0 & 0.118854 & 3.081042 & -0.249991 \\ 14 & 7 & 0 & -2.662202 & -0.765848 & -0.266579 \\ 15 & 1 & 0 & -2.214317 & 1.278969 & -0.033798 \\ 16 & 1 & 0 & -1.725026 & -0.771251 & 2.274293 \\ 17 & 1 & 0 & -1.537202 & 1.071807 & 2.417231 \\ 18 & 1 & 0 & 2.344453 & -1.109707 & -0.879262 \\ 19 & 7 & 0 & 0.748746 & -1.135890 & -2.250053 \\ 20 & 1 & 0 & 1.080432 & -2.287462 & 0.982498 \\ 21 & 1 & 0 & -0.436750 & -2.323476 & -0.076132 \\ 22 & 8 & 0 & 1.573297 & -0.708683 & -3.144497 \\ 23 & 8 & 0 & -0.472745 & -1.442435 & -2.495538 \\ 24 & 8 & 0 & -3.308198 & -0.448884 & -1.320644 \\ 25 & 8 & 0 & -2.618286 & -1.951341 & 0.221581\end{array}$

$\begin{array}{llll}\text { Maximum Force } & 0.000016 & 0.000450 & \text { YES } \\ \text { RMS Force } & 0.000003 & 0.000300 & \text { YES } \\ \text { Maximum Displacement } & 0.001738 & 0.001800 & \text { YES } \\ \text { RMS Displacement } & 0.000442 & 0.001200 & \text { YES } \\ \text { Predicted change in Energy=-1.323507D-08 } & \end{array}$

SCF Done: $E(R B+H F-L Y P)=-880.954018483 \quad$ A.U. after 14 cycles Convg $=0.7944 \mathrm{D}-08 \quad \mathrm{~S} * * 2=0.0000$

\section{$5 \cdot \mathrm{C}_{2} \mathrm{H}_{3} \mathrm{NO}_{2}-\mathrm{J}$}

\begin{tabular}{|c|c|c|c|c|c|}
\hline \multirow{3}{*}{$\begin{array}{l}\text { Center } \\
\text { Number } \\
------ \\
1\end{array}$} & \multirow{2}{*}{\multicolumn{2}{|c|}{$\begin{array}{l}\text { Atomic } \\
\text { Number }\end{array}$}} & \multicolumn{3}{|c|}{ Coordinates (Angstroms) } \\
\hline & & & X & $\mathrm{Y}$ & $\mathrm{Z}$ \\
\hline & 6 & 0 & -1.847058 & 0.326115 & -0.356318 \\
\hline 2 & 6 & 0 & -1.852116 & 0.354580 & 1.049234 \\
\hline 3 & 46 & 0 & 0.282833 & 0.316977 & 0.115599 \\
\hline 4 & 6 & 0 & 0.498551 & -1.831009 & -0.195653 \\
\hline 5 & 6 & 0 & 1.002395 & -1.303935 & -1.397572 \\
\hline 6 & 7 & 0 & 2.117214 & 0.663620 & 1.235527 \\
\hline 7 & 6 & 0 & 2.573673 & 1.898329 & 1.196464 \\
\hline 8 & 6 & 0 & 1.718877 & 2.896169 & 0.563842 \\
\hline 9 & 7 & 0 & 0.670552 & 2.448313 & -0.095671 \\
\hline 10 & 1 & 0 & 2.736937 & -0.031524 & 1.666543 \\
\hline 11 & 1 & 0 & 3.540261 & 2.196092 & 1.603851 \\
\hline 12 & 1 & 0 & 1.965848 & 3.954293 & 0.654873 \\
\hline 13 & 1 & 0 & 0.069476 & 3.162100 & -0.522588 \\
\hline 14 & 1 & 0 & -2.076153 & -0.588312 & -0.897060 \\
\hline
\end{tabular}




$\begin{array}{rrrrrr}15 & 1 & 0 & -2.017770 & 1.257276 & -0.891772 \\ 16 & 7 & 0 & -2.097699 & -0.835009 & 1.844490 \\ 17 & 1 & 0 & -1.933329 & 1.251339 & 1.651180 \\ 18 & 1 & 0 & 2.049882 & -1.122253 & -1.604587 \\ 19 & 7 & 0 & 0.212973 & -1.269634 & -2.615539 \\ 20 & 1 & 0 & 1.207795 & -2.160036 & 0.560449 \\ 21 & 1 & 0 & -0.491044 & -2.278132 & -0.153076 \\ 22 & 8 & 0 & -2.144519 & -0.678527 & 3.122866 \\ 23 & 8 & 0 & -2.267911 & -1.972071 & 1.263962 \\ 24 & 8 & 0 & 0.799346 & -0.827109 & -3.674465 \\ 25 & 8 & 0 & -1.005594 & -1.686578 & -2.603238 \\ ---------------------------------------------------------------------\end{array}$

$\begin{array}{lccc}\text { Maximum Force } & 0.000374 & 0.000450 & \text { YES } \\ \text { RMS Force } & 0.000118 & 0.000300 & \text { YES } \\ \text { Maximum Displacement } & 0.000696 & 0.001800 & \text { YES } \\ \text { RMS Displacement } & 0.000144 & 0.001200 & \text { YES } \\ \text { Predicted change in Energy }=-1.374524 D-06 & \end{array}$

SCF Done: $\mathrm{E}(\mathrm{RB}+\mathrm{HF}-\mathrm{LYP})=-880.966049399$ A.U. after 20 cycles Convg $=0.8072 \mathrm{D}-08 \quad \mathrm{~S} * * 2=0.0000$

\section{$\underline{5-\mathrm{CH}_{2}} \underline{\mathrm{Cl}}_{2}$}

\begin{tabular}{|c|c|c|c|c|c|}
\hline \multirow{2}{*}{$\begin{array}{l}\text { Center } \\
\text { Number }\end{array}$} & \multirow{2}{*}{\multicolumn{2}{|c|}{$\begin{array}{l}\text { Atomic } \\
\text { Number }\end{array}$}} & \multicolumn{3}{|c|}{ Coordinates (Angstroms) } \\
\hline & & & X & $\mathrm{Y} \quad \mathrm{Z}$ & $\mathrm{Z}$ \\
\hline 1 & 6 & 0 & 1.243120 & -1.631891 & -0.32365 \\
\hline 2 & 46 & & -0.307306 & -0.242017 & $7 \quad-0.169969$ \\
\hline 3 & 7 & 0 & -2.254713 & -0.981615 & 0.355083 \\
\hline 4 & 6 & & .216971 & -0.105228 & 0.407 \\
\hline 5 & 6 & & -2.850016 & 1.297808 & 0.115822 \\
\hline 6 & 7 & 0 & -1.598501 & 1.522900 & -0.16 \\
\hline 7 & 6 & 0 & 1.777983 & -0.348728 & -0.703559 \\
\hline 8 & 7 & 0 & 631937 & 0.389904 & 0.200844 \\
\hline 9 & 8 & 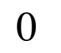 & & -0.00 & \\
\hline 10 & 8 & & 3.216887 & 1.450312 & -0.252973 \\
\hline 11 & 1 & 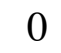 & -2.500450 & -1.957283 & 0.558633 \\
\hline 12 & 1 & 0 & -4.250675 & -0.354059 & 0.651153 \\
\hline 13 & 1 & 0 & -3.618422 & 2.071538 & 0.145356 \\
\hline 14 & 1 & & -1.338482 & 2.495690 & -0.367776 \\
\hline 15 & 1 & & 1.519123 & -2.051872 & 0.641564 \\
\hline 16 & 1 & 0 & 1.069783 & -2.356911 & -1.117717 \\
\hline 17 & 1 & 0 & 1.960215 & -0.056428 & -1.734587 \\
\hline
\end{tabular}




$\begin{array}{lccc}\text { Maximum Force } & 0.000004 & 0.000002 & \text { NO } \\ \text { RMS Force } & 0.000001 & 0.000001 & \text { YES } \\ \text { Maximum Displacement } & 0.009203 & 0.000006 & \text { NO } \\ \text { RMS Displacement } & 0.002039 & 0.000004 & \text { NO } \\ \text { Predicted change in Energy=-1.303636D-05 } & \end{array}$

SCF Done: $\mathrm{E}(\mathrm{RB}+\mathrm{HF}-\mathrm{LYP})=-597.928307052$ A.U. after 8 cycles Convg $=0.8911 \mathrm{D}-08 \quad \mathrm{~S} * * 2=0.0000$

\section{$\underline{\mathrm{C}}_{2} \underline{\mathrm{H}}_{3} \underline{\mathrm{NO}_{2}}-\mathrm{CH}_{2} \underline{\mathrm{Cl}}_{2}$}

\begin{tabular}{|c|c|c|c|c|c|}
\hline \multirow{2}{*}{$\begin{array}{l}\text { Center } \\
\text { Number }\end{array}$} & \multirow{2}{*}{\multicolumn{2}{|c|}{$\begin{array}{l}\text { Atomic } \\
\text { Number }\end{array}$}} & \multicolumn{3}{|c|}{ Coordinates (Angstroms) } \\
\hline & & & $\mathrm{X}$ & $\mathrm{Y}$ & $\mathrm{Z}$ \\
\hline 1 & 6 & 0 & -1.898191 & 0.037681 & -0.000276 \\
\hline 2 & 6 & 0 & -0.748809 & -0.655713 & 0.000317 \\
\hline 3 & 7 & 0 & 0.547311 & 0.025729 & -0.000081 \\
\hline 4 & 8 & 0 & 1.586034 & -0.725171 & -0.000259 \\
\hline 5 & 8 & 0 & 0.595498 & 1.303747 & 0.000269 \\
\hline 6 & 1 & 0 & -1.907209 & 1.124090 & -0.001001 \\
\hline 7 & 1 & 0 & -2.846922 & -0.490351 & 0.000231 \\
\hline 8 & 1 & 0 & -0.647296 & -1.734261 & 0.001008 \\
\hline
\end{tabular}

$\begin{array}{lccc}\text { Maximum Force } & 0.000049 & 0.000450 & \text { YES } \\ \text { RMS Force } & 0.000029 & 0.000300 & \text { YES } \\ \text { Maximum Displacement } & 0.000431 & 0.001800 & \text { YES } \\ \text { RMS Displacement } & 0.000166 & 0.001200 & \text { YES }\end{array}$

Predicted change in Energy=-4.471457D-08

SCF Done: $\mathrm{E}(\mathrm{RB}+\mathrm{HF}-\mathrm{LYP})=-283.054608506$ A.U. after 6 cycles Convg $=0.5969 \mathrm{D}-08 \quad \mathrm{~S} * * 2=0.0000$

$\left[5 / \mathrm{C}_{2} \underline{\mathrm{H}}_{3} \underline{\mathrm{NO}_{2}}\right]^{*}-\mathrm{CH}_{2} \underline{\mathrm{Cl}}_{2}$

\begin{tabular}{|c|c|c|c|c|}
\hline \multirow{2}{*}{$\begin{array}{l}\text { Center } \\
\text { Number }\end{array}$} & \multirow{2}{*}{$\begin{array}{l}\text { Atomic } \\
\text { Number }\end{array}$} & \multicolumn{3}{|c|}{ Coordinates (Angstroms) } \\
\hline & & $\mathrm{X}$ & $\mathrm{Y}$ & $\mathrm{Z}$ \\
\hline 1 & 6 & -1.833423 & -0.746865 & -1.526144 \\
\hline 2 & 6 & -2.244088 & -1.008667 & -0.183624 \\
\hline 3 & 7 & -3.266470 & -0.187789 & 0.446780 \\
\hline 4 & 8 & -3.695645 & 0.878319 & -0.133767 \\
\hline 5 & 46 & -0.328438 & 0.039354 & -0.269607 \\
\hline
\end{tabular}




$\begin{array}{cccccc}6 & 6 & 0 & 0.763206 & -1.990866 & 0.080881 \\ 7 & 6 & 0 & 1.925918 & -1.573831 & -0.540538 \\ 8 & 7 & 0 & 2.993423 & -0.972596 & 0.179383 \\ 9 & 8 & 0 & 4.011218 & -0.543756 & -0.503172 \\ 10 & 7 & 0 & 0.717082 & 1.799266 & -1.207454 \\ 11 & 6 & 0 & 1.370197 & 2.546294 & -0.366337 \\ 12 & 6 & 0 & 1.401086 & 2.113644 & 1.051467 \\ 13 & 7 & 0 & 0.731646 & 1.045495 & 1.366902 \\ 14 & 8 & 0 & -3.720219 & -0.580389 & 1.587730 \\ 15 & 8 & 0 & 2.933817 & -0.854710 & 1.468583 \\ 16 & 1 & 0 & -2.183209 & -1.980167 & 0.299247 \\ 17 & 1 & 0 & -1.460634 & -1.578261 & -2.121161 \\ 18 & 1 & 0 & -2.312459 & 0.056232 & -2.080283 \\ 19 & 1 & 0 & 0.793450 & 0.736988 & 2.344344 \\ 20 & 1 & 0 & 1.986672 & 2.692618 & 1.766857 \\ 21 & 1 & 0 & 1.897560 & 3.459563 & -0.648833 \\ 22 & 1 & 0 & 0.711810 & 2.104076 & -2.189678 \\ 23 & 1 & 0 & 0.699804 & -2.027719 & 1.164655 \\ 24 & 1 & 0 & 0.092564 & -2.634808 & -0.480485 \\ 25 & 1 & 0 & 2.122076 & -1.663411 & -1.601225\end{array}$

$\begin{array}{llll}\text { Maximum Force } & 0.000431 & 0.000450 & \text { YES } \\ \text { RMS Force } & 0.000129 & 0.000300 & \text { YES } \\ \text { Maximum Displacement } & 0.007600 & 0.001800 & \text { NO } \\ \text { RMS Displacement } & 0.001490 & 0.001200 & \text { NO } \\ \text { Predicted change in Energy }=1.987901 D-08 & \end{array}$

Predicted change in Energy= 1.987901D-08

SCF Done: $\mathrm{E}(\mathrm{RB}+\mathrm{HF}-\mathrm{LYP})=-880.984521643$ A.U. after 10 cycles Convg $=0.9326 \mathrm{D}-08 \quad \mathrm{~S} * * 2=0.0000$

\section{$\underline{5 \cdot \mathrm{C}_{2}} \underline{\mathrm{H}}_{2} \underline{\mathrm{NO}}_{2}-\mathrm{CH}_{2} \mathrm{Cl}_{2}$}

\begin{tabular}{|c|c|c|c|c|c|}
\hline \multirow{2}{*}{$\begin{array}{l}\text { Center } \\
\text { Number }\end{array}$} & \multirow{2}{*}{\multicolumn{2}{|c|}{$\begin{array}{l}\text { Atomic } \\
\text { Number }\end{array}$}} & \multirow{2}{*}{$\begin{array}{l}\text { Atomic } \\
\text { Type }\end{array}$} & \multicolumn{2}{|c|}{ Coordinates (Angstrom } \\
\hline & & & & X $\quad \mathrm{Y}$ & $\mathrm{Z}$ \\
\hline 1 & 6 & 0 & -1.327435 & -1.696882 & -0.840508 \\
\hline 2 & 46 & 0 & -0.001556 & -0.249179 & -0.007931 \\
\hline 3 & 7 & 0 & -0.867532 & 1.501777 & -1.012274 \\
\hline 4 & 6 & 0 & -0.525552 & 2.653070 & -0.510033 \\
\hline 5 & 6 & 0 & 0.542369 & 2.646547 & 0.511099 \\
\hline 6 & 7 & 0 & 0.871472 & 1.490565 & 1.011016 \\
\hline 7 & 6 & 0 & -2.058834 & -1.280204 & 0.302795 \\
\hline 8 & 7 & 0 & -3.147750 & -0.358735 & 0.187860 \\
\hline 9 & 8 & 0 & -3.406784 & 0.209612 & -0.952293 \\
\hline
\end{tabular}




$\begin{array}{rrrrrr}10 & 6 & 0 & 1.309958 & -1.736713 & 0.778673 \\ 11 & 6 & 0 & 2.061338 & -1.272383 & -0.332283 \\ 12 & 7 & 0 & 3.150290 & -0.360420 & -0.157942 \\ 13 & 8 & 0 & 3.390950 & 0.155994 & 1.010599 \\ 14 & 8 & 0 & -3.860102 & -0.127051 & 1.242215 \\ 15 & 8 & 0 & 3.880915 & -0.083561 & -1.188780 \\ 16 & 1 & 0 & 1.650497 & 1.464232 & 1.679797 \\ 17 & 1 & 0 & 1.011285 & 3.587552 & 0.802656 \\ 18 & 1 & 0 & -0.990773 & 3.598645 & -0.792635 \\ 19 & 1 & 0 & -1.659019 & 1.484664 & -1.666575 \\ 20 & 1 & 0 & -0.847920 & -2.671750 & -0.808943 \\ 21 & 1 & 0 & -1.641051 & -1.355147 & -1.823341 \\ 22 & 1 & 0 & -2.039035 & -1.789402 & 1.261922 \\ 23 & 1 & 0 & 2.056594 & -1.737651 & -1.313858 \\ 24 & 1 & 0 & 1.604596 & -1.443766 & 1.782884 \\ 25 & 1 & 0 & 0.830146 & -2.708015 & 0.689915 \\ ---------------------------------------------------------------------\end{array}$

$\begin{array}{llll}\text { Maximum Force } & 0.000355 & 0.000450 & \text { YES } \\ \text { RMS Force } & 0.000122 & 0.000300 & \text { YES }\end{array}$

Maximum Displacement $\quad 0.000325 \quad 0.001800 \quad$ YES

RMS Displacement $0.000099 \quad 0.001200 \quad$ YES

Predicted change in Energy=-7.333028D-05

SCF Done: $\mathrm{E}(\mathrm{RB}+\mathrm{HF}-\mathrm{LYP})=-880.995206267$ A.U. after 6 cycles Convg $=0.5805 \mathrm{D}-08 \quad \mathrm{~S} * * 2=0.0000$

\section{$\underline{5 \text { - toluene }}$}

\begin{tabular}{|c|c|c|c|c|c|}
\hline \multirow{2}{*}{$\begin{array}{l}\text { Center } \\
\text { Number }\end{array}$} & \multirow{2}{*}{\multicolumn{2}{|c|}{$\begin{array}{l}\text { Atomic } \\
\text { Number }\end{array}$}} & \multicolumn{3}{|c|}{ Coordinates (Angstroms) } \\
\hline & & & X & $\mathrm{Y}$ & Z \\
\hline 1 & 6 & 0 & 1.261386 & -1.648493 & -0.286000 \\
\hline 2 & 46 & & & -0.264785 & -0.167969 \\
\hline 3 & 7 & 0 & -2.276905 & -0.961011 & \\
\hline 4 & 6 & & 29 & -0.06 & \\
\hline 5 & 6 & 0 & -2.820704 & 1.328706 & 0.110069 \\
\hline 6 & 7 & 0 & -1.564917 & 1.519507 & -0.180022 \\
\hline 7 & 6 & 0 & 1.769862 & -0.365345 & -0.691150 \\
\hline 8 & 7 & 0 & & 0.409109 & 0.199503 \\
\hline 9 & 8 & 0 & & 0.04 & 1.423 \\
\hline 10 & 8 & 0 & 64 & 1.458615 & -0.289540 \\
\hline 11 & 1 & 0 & -2.546751 & -1.928343 & 0.551810 \\
\hline 12 & 1 & 0 & -4.262685 & -0.288673 & 0.631371 \\
\hline 13 & 1 & 0 & -3.567110 & 2.122498 & 0.153970 \\
\hline
\end{tabular}




$\begin{array}{rrrrrr}14 & 1 & 0 & -1.274987 & 2.483215 & -0.373168 \\ 15 & 1 & 0 & 1.537244 & -2.035687 & 0.692185 \\ 16 & 1 & 0 & 1.099712 & -2.395470 & -1.060844 \\ 17 & 1 & 0 & 1.959405 & -0.094699 & -1.722136\end{array}$

$\begin{array}{lccc}\text { Maximum Force } & 0.000042 & 0.000002 & \text { NO } \\ \text { RMS Force } & 0.000016 & 0.000001 & \text { NO } \\ \text { Maximum Displacement } & 0.007882 & 0.000006 & \text { NO } \\ \text { RMS Displacement } & 0.001714 & 0.000004 & \text { NO } \\ \text { Predicted change in Energy=-3.583926D-07 } & \end{array}$

SCF Done: $\mathrm{E}(\mathrm{RB}+\mathrm{HF}-\mathrm{LYP})=-597.918878714$ A.U. after 8 cycles Convg $=0.9862 \mathrm{D}-08 \quad \mathrm{~S} * * 2=0.0000$

\section{$\underline{\mathrm{C}}_{2} \underline{\mathrm{H}}_{3} \underline{\mathrm{NO}_{2}} \underline{\text { - toluene }}$}

\begin{tabular}{|c|c|c|c|c|c|}
\hline \multirow{2}{*}{$\begin{array}{l}\text { Center } \\
\text { Number }\end{array}$} & \multirow{2}{*}{\multicolumn{2}{|c|}{$\begin{array}{l}\text { Atomic } \\
\text { Number }\end{array}$}} & \multicolumn{3}{|c|}{ Coordinates (Angstroms) } \\
\hline & & & $\mathrm{X}$ & $\mathrm{Y}$ & $\mathrm{Z}$ \\
\hline 1 & 6 & 0 & -1.896939 & 0.035233 & 0.000361 \\
\hline 2 & 6 & 0 & -0.747650 & -0.657019 & -0.000517 \\
\hline 3 & 7 & 0 & 0.549266 & 0.027558 & -0.000112 \\
\hline 4 & 8 & 0 & 1.585894 & -0.724664 & 0.000491 \\
\hline 5 & 8 & 0 & 0.590695 & 1.304769 & -0.000310 \\
\hline 6 & 1 & 0 & -1.901826 & 1.121447 & 0.001460 \\
\hline 7 & 1 & 0 & -2.846537 & -0.490407 & 0.000238 \\
\hline 8 & 1 & 0 & -0.641677 & -1.734072 & -0.001424 \\
\hline
\end{tabular}

$\begin{array}{llll}\text { Maximum Force } & 0.000156 & 0.000450 & \text { YES } \\ \text { RMS Force } & 0.000062 & 0.000300 & \text { YES } \\ \text { Maximum Displacement } & 0.001741 & 0.001800 & \text { YES } \\ \text { RMS Displacement } & 0.000600 & 0.001200 & \text { YES } \\ \text { Predicted change in Energy=-1.806477D-07 } & \end{array}$

SCF Done: $\mathrm{E}(\mathrm{RB}+\mathrm{HF}-\mathrm{LYP})=-283.052087919$ A.U. after 7 cycles Convg $=0.2142 \mathrm{D}-08 \quad \mathrm{~S} * * 2=0.0000$

$\left[5 / \mathrm{C}_{2} \underline{H}_{3} \underline{N_{2} O_{2}}\right]^{\sharp}$ - toluene

Center Atomic Coordinates (Angstroms)

Number Number $\quad$ X $\quad$ Y $\quad$ Z 


$\begin{array}{cccccc}1 & 6 & 0 & -1.885549 & -0.635367 & -1.554441 \\ 2 & 6 & 0 & -2.244065 & -0.977246 & -0.216623 \\ 3 & 7 & 0 & -3.248369 & -0.196934 & 0.499554 \\ 4 & 8 & 0 & -3.683447 & 0.905333 & 0.002925 \\ 5 & 46 & 0 & -0.330876 & 0.057861 & -0.297936 \\ 6 & 6 & 0 & 0.747713 & -2.008013 & -0.095405 \\ 7 & 6 & 0 & 1.920797 & -1.527562 & -0.644684 \\ 8 & 7 & 0 & 2.961832 & -0.988271 & 0.164243 \\ 9 & 8 & 0 & 3.968167 & -0.443362 & -0.442887 \\ 10 & 7 & 0 & 0.678202 & 1.894078 & -1.121923 \\ 11 & 6 & 0 & 1.376890 & 2.564500 & -0.251910 \\ 12 & 6 & 0 & 1.487577 & 2.001426 & 1.112902 \\ 13 & 7 & 0 & 0.807438 & 0.924387 & 1.372336 \\ 14 & 8 & 0 & -3.661466 & -0.668306 & 1.623425 \\ 15 & 8 & 0 & 2.877757 & -1.038028 & 1.455816 \\ 16 & 1 & 0 & -2.173299 & -1.973577 & 0.201324 \\ 17 & 1 & 0 & -1.539886 & -1.425713 & -2.216739 \\ 18 & 1 & 0 & -2.383490 & 0.202928 & -2.033810 \\ 19 & 1 & 0 & 0.941431 & 0.514652 & 2.301765 \\ 20 & 1 & 0 & 2.143603 & 2.490036 & 1.833554 \\ 21 & 1 & 0 & 1.889467 & 3.501054 & -0.477288 \\ 22 & 1 & 0 & 0.619302 & 2.289963 & -2.065857 \\ 23 & 1 & 0 & 0.666807 & -2.145065 & 0.978409 \\ 24 & 1 & 0 & 0.089046 & -2.595750 & -0.726579 \\ 25 & 1 & 0 & 2.145322 & -1.504472 & -1.702465 \\ ---------------------------------------------------------\end{array}$

$\begin{array}{llll}\text { Maximum Force } & 0.000164 & 0.000450 & \text { YES } \\ \text { RMS Force } & 0.000051 & 0.000300 & \text { YES } \\ \text { Maximum Displacement } & 0.009624 & 0.001800 & \text { NO } \\ \text { RMS Displacement } & 0.002164 & 0.001200 & \text { NO } \\ \text { Predicted change in Energy= }=8.914406 \mathrm{D}-08 & \end{array}$

SCF Done: $\mathrm{E}(\mathrm{RB}+\mathrm{HF}-\mathrm{LYP})=-880.973605398$ A.U. after 10 cycles Convg $=0.9944 \mathrm{D}-08 \quad \mathrm{~S} * * 2=0.0000$

\section{$\underline{5}_{2} \underline{\mathrm{H}}_{3} \underline{\mathrm{NO}}_{2}$ - toluene}

\begin{tabular}{lccccc} 
Center & \multicolumn{2}{c}{ Atomic } & \multicolumn{3}{c}{ Coordinates (Angstroms) } \\
Number & Number & \multicolumn{2}{c}{ X } & Y & Z \\
--1 & 6 & 0 & -1.351334 & -1.747284 & -0.776168 \\
2 & 46 & 0 & 0.000004 & -0.272325 & -0.000008 \\
3 & 6 & 0 & 1.351346 & -1.747271 & 0.776172
\end{tabular}




$\begin{array}{cccccc}4 & 6 & 0 & 2.075371 & -1.260693 & -0.340528 \\ 5 & 7 & 0 & 3.159299 & -0.321473 & -0.174657 \\ 6 & 8 & 0 & 3.406235 & 0.177244 & 0.998205 \\ 7 & 6 & 0 & -2.075354 & -1.260705 & 0.340537 \\ 8 & 7 & 0 & -3.159289 & -0.321495 & 0.174670 \\ 9 & 8 & 0 & -3.406238 & 0.177215 & -0.998192 \\ 10 & 8 & 0 & -3.849413 & -0.020139 & 1.217333 \\ 11 & 7 & 0 & -0.896900 & 1.481593 & -0.983229 \\ 12 & 6 & 0 & -0.560425 & 2.636053 & -0.479646 \\ 13 & 6 & 0 & 0.560372 & 2.636058 & 0.479644 \\ 14 & 7 & 0 & 0.896868 & 1.481597 & 0.983214 \\ 15 & 8 & 0 & 3.849442 & -0.020133 & -1.217313 \\ 16 & 1 & 0 & 1.731533 & 1.442130 & 1.580183 \\ 17 & 1 & 0 & 1.068487 & 3.571805 & 0.717478 \\ 18 & 1 & 0 & -1.068554 & 3.571797 & -0.717462 \\ 19 & 1 & 0 & -1.731567 & 1.442131 & -1.580196 \\ 20 & 1 & 0 & -0.885297 & -2.725942 & -0.700180 \\ 21 & 1 & 0 & -1.660163 & -1.442475 & -1.772480 \\ 22 & 1 & 0 & -2.090658 & -1.728065 & 1.317226 \\ 23 & 1 & 0 & 2.090690 & -1.728045 & -1.317221 \\ 24 & 1 & 0 & 1.660153 & -1.442451 & 1.772486 \\ 25 & 1 & 0 & 0.885309 & -2.725931 & 0.700204 \\ & 1 & & & \end{array}$

$\begin{array}{llll}\text { Maximum Force } & 0.000000 & 0.000002 & \text { YES } \\ \text { RMS Force } & 0.000000 & 0.000001 & \text { YES } \\ \text { Maximum Displacement } & 0.000003 & 0.000006 & \text { YES } \\ \text { RMS Displacement } & 0.000001 & 0.000004 & \text { YES } \\ \text { Predicted change in Energy=-6.687837D-14 } & \end{array}$

SCF Done: $\mathrm{E}(\mathrm{RB}+\mathrm{HF}-\mathrm{LYP})=-880.985533590 \quad$ A.U. after 6 cycles Convg $=0.5980 \mathrm{D}-08 \quad \mathrm{~S} * * 2=0.0000$ 
Gaussian98 Results (xyz cooridinates, optimization gradients, total energy) for the SelfExchange of $\mathrm{C}_{2} \mathrm{H}_{4}$ with 4 using the Stuttgart RSC 1997 ECP basis set (Basis B).

\section{4-gas phase}

\begin{tabular}{|c|c|c|c|c|c|}
\hline \multirow{2}{*}{$\begin{array}{l}\text { Center } \\
\text { Number }\end{array}$} & \multirow{2}{*}{\multicolumn{2}{|c|}{$\begin{array}{l}\text { Atomic } \\
\text { Number }\end{array}$}} & \multicolumn{3}{|c|}{ Coordinates (Angstroms) } \\
\hline & & & $\mathrm{X}$ & Y & $\mathrm{Z}$ \\
\hline 1 & 6 & 0 & 2.416824 & 0.706639 & 0.034010 \\
\hline 2 & 46 & 0 & .427079 & -0.000258 & -0.018305 \\
\hline 3 & 7 & 0 & -1.373093 & 1.314965 & -0.009403 \\
\hline 4 & 6 & 0 & -2.517092 & 0.733720 & 0.026568 \\
\hline 5 & 6 & 0 & -2.515747 & -0.734970 & 0.026231 \\
\hline 6 & 7 & 0 & -1.370593 & -1.313962 & -0.009308 \\
\hline 7 & 6 & 0 & 2.417606 & -0.704886 & 0.034583 \\
\hline 8 & 1 & 0 & -1.427140 & 2.331124 & -0.010848 \\
\hline 9 & 1 & 0 & 3 & 1.265938 & \\
\hline 10 & 1 & 0 & -3.466 & -1.269055 & 0.054271 \\
\hline 11 & 1 & 0 & -1.422527 & -2.330220 & -0.01144 \\
\hline 12 & 1 & 0 & 2.655879 & 1.253596 & -0.874585 \\
\hline 13 & 1 & 0 & 2.610142 & 1.255351 & 0.952198 \\
\hline 14 & 1 & 0 & & -1.2 & -0.87 \\
\hline 15 & 1 & 0 & 2.611604 & -1.252592 & 0.953239 \\
\hline \multicolumn{3}{|c|}{ Maximum Force } & 0.00016 & \multirow{2}{*}{$\begin{array}{l}0.000450 \\
0.000300\end{array}$} & YES \\
\hline \multirow{2}{*}{\multicolumn{3}{|c|}{$\begin{array}{l}\text { RMS Force } \\
\text { Maximum Disp }\end{array}$}} & 0.00002 & & YES \\
\hline & & & 0.00155 & 0.001800 & YES \\
\hline RMS 1 & \multicolumn{2}{|c|}{ Displacement } & 0.00038 & 0.0012 & YES \\
\hline
\end{tabular}

SCF Done: $\mathrm{E}(\mathrm{RB}+\mathrm{HF}-\mathrm{LYP})=-394.730793585 \quad$ A.U. after 39 cycles Convg $=0.9066 \mathrm{D}-08 \quad \mathrm{~S} * * 2=0.0000$

\section{$\underline{\mathbf{C}}_{2} \underline{H}_{4}-$ gas phase}

\begin{tabular}{llllll} 
Center & Atomic & & \multicolumn{3}{c}{ Coordinates (Angstroms) } \\
Number & Number & X & Y & Z \\
\hline 1 & 6 & 0 & 0.000000 & 0.000000 & -0.664505 \\
2 & 6 & 0 & 0.000000 & 0.000000 & 0.664505 \\
3 & 1 & 0 & 0.923106 & 0.000000 & 1.234514
\end{tabular}




$\begin{array}{rrrrrr}4 & 1 & 0 & 0.923106 & 0.000000 & -1.234514 \\ 5 & 1 & 0 & -0.923106 & 0.000000 & 1.234514 \\ 6 & 1 & 0 & -0.923106 & 0.000000 & -1.234514\end{array}$

$\begin{array}{lccc}\text { Maximum Force } & 0.000158 & 0.000450 & \text { YES } \\ \text { RMS Force } & 0.000080 & 0.000300 & \text { YES } \\ \text { Maximum Displacement } & 0.001107 & 0.001800 & \text { YES } \\ \text { RMS Displacement } & 0.000630 & 0.001200 & \text { YES } \\ \text { Predicted change in Energy }=-3.228248 D-07 & \end{array}$

SCF Done: $\mathrm{E}(\mathrm{RB}+\mathrm{HF}-\mathrm{LYP})=-78.6155381588 \quad$ A.U. after 1 cycles Convg $=0.1332 \mathrm{D}-09 \quad \mathrm{~S} * * 2=0.0000$

\section{$\left[4 / \mathrm{C}_{2} \underline{\mathrm{H}_{1}}\right]^{\ddagger}$ - gas phase}

\begin{tabular}{|c|c|c|c|c|c|}
\hline \multirow{2}{*}{$\begin{array}{l}\text { Center } \\
\text { Number }\end{array}$} & \multirow{2}{*}{\multicolumn{2}{|c|}{$\begin{array}{l}\text { Atomic } \\
\text { Number }\end{array}$}} & \multicolumn{3}{|c|}{ Coordinates (Angstroms) } \\
\hline & & & X & $\mathrm{Y}$ & $\mathrm{Z}$ \\
\hline 1 & 46 & 0 & -0.211029 & -0.275645 & -0.05745 \\
\hline 2 & 7 & 0 & 1.447311 & 0.673548 & -1.174083 \\
\hline 3 & 6 & 0 & 2.623057 & 0.530115 & -0.644732 \\
\hline 4 & 6 & 0 & 2.713668 & -0.267753 & 0.559669 \\
\hline 5 & 7 & 0 & 1.597749 & -0.538922 & 1.168024 \\
\hline 6 & 1 & 0 & 1.443185 & 1.303095 & -1.974246 \\
\hline 7 & 1 & 0 & 3.523518 & 0.982592 & -1.059977 \\
\hline 8 & 1 & 0 & 3.686794 & -0.613330 & 0.907352 \\
\hline 9 & 1 & 0 & 1.720716 & -1.131124 & 1.986651 \\
\hline 10 & 6 & 0 & & -1.639577 & 0.50403 \\
\hline 11 & 6 & 0 & 87 & -1.476143 & -0.88925 \\
\hline 12 & 1 & 0 & -2.478921 & -1.146041 & 1.13727 \\
\hline 13 & 1 & 0 & -1.305231 & -2.533671 & $0.93524 \varepsilon$ \\
\hline 14 & 1 & 0 & -2.553941 & -0.849368 & -1.34546 \\
\hline 15 & 1 & 0 & -1.364984 & -2.223820 & -1.54844 \\
\hline 16 & 6 & 0 & -1.259183 & 2.278741 & 0.991132 \\
\hline 17 & 6 & 0 & -1.869628 & 2.099242 & -0.183460 \\
\hline 18 & 1 & 0 & -0.313325 & 2.802702 & 1.068297 \\
\hline 19 & 1 & 0 & -1.689818 & 1.916828 & 1.917804 \\
\hline 20 & 1 & 0 & & 2.483535 & -1.108128 \\
\hline 21 & 1 & 0 & -2.828025 & 1.598153 & -0.255530 \\
\hline & & & 0.000 & 0.00045 & YES \\
\hline & & & & 0.00050 & YES \\
\hline Maximu & & & 0.00315 & 0.00180 & $\mathrm{NO}$ \\
\hline
\end{tabular}


RMS Displacement $0.0006890 .001200 \quad$ YES Predicted change in Energy=-5.193927D-09

SCF Done: $\mathrm{E}(\mathrm{RB}+\mathrm{HF}-\mathrm{LYP})=-473.341763107$ A.U. after 2 cycles Convg $=0.1919 \mathrm{D}-08 \quad \mathrm{~S} * * 2=0.0000$

\section{$\underline{4 \cdot \mathbf{C}_{2}} \underline{\mathbf{H}_{4}}$ - gas phase}

\begin{tabular}{cccccc} 
Center & Atomic & \multicolumn{4}{c}{ Coordinates (Angstroms) } \\
Number & Number & \multicolumn{2}{c}{ X } & Y & $Z$ \\
---------------- \\
1 & 46 & 0 & 0.243852 & -0.000351 & 0.000303 \\
2 & 6 & 0 & 1.355524 & -1.746259 & 0.966263 \\
3 & 6 & 0 & 1.756316 & -1.542899 & -0.344495 \\
4 & 6 & 0 & 1.752187 & 1.547314 & 0.341657 \\
5 & 6 & 0 & 1.346538 & 1.750083 & -0.967648 \\
6 & 7 & 0 & -1.449291 & 1.090790 & 0.729366 \\
7 & 6 & 0 & -2.612874 & 0.642591 & 0.299289 \\
8 & 6 & 0 & -2.613072 & -0.643518 & -0.301926 \\
9 & 7 & 0 & -1.448757 & -1.096067 & -0.725409 \\
10 & 1 & 0 & 0.617622 & -2.497421 & 1.217669 \\
11 & 1 & 0 & 1.921004 & -1.339996 & 1.795709 \\
12 & 1 & 0 & 1.375571 & -2.173875 & -1.140403 \\
13 & 1 & 0 & 2.695717 & -1.047552 & -0.563744 \\
14 & 1 & 0 & 2.694398 & 1.055432 & 0.556725 \\
15 & 1 & 0 & 1.371625 & 2.175379 & 1.139918 \\
16 & 1 & 0 & 0.604793 & 2.498383 & -1.216355 \\
17 & 1 & 0 & 1.911267 & 1.346672 & -1.798993 \\
18 & 1 & 0 & -1.485413 & 2.056762 & 1.048192 \\
19 & 1 & 0 & -3.529470 & 1.221246 & 0.385702 \\
20 & 1 & 0 & -3.530251 & -1.221272 & -0.388358 \\
21 & 1 & 0 & -1.485432 & -2.064559 & -1.036556
\end{tabular}

$\begin{array}{lccc}\text { Maximum Force } & 0.000003 & 0.000450 & \text { YES } \\ \text { RMS Force } & 0.000001 & 0.000300 & \text { YES } \\ \text { Maximum Displacement } & 0.004151 & 0.001800 & \text { NO } \\ \text { RMS Displacement } & 0.001206 & 0.001200 & \text { NO }\end{array}$

Predicted change in Energy=-1.056969D-07

SCF Done: $\mathrm{E}(\mathrm{RB}+\mathrm{HF}-\mathrm{LYP})=-473.349626871 \quad$ A.U. after 33 cycles Convg $=0.8681 \mathrm{D}-08 \quad \mathrm{~S} * * 2=0.0000$

\section{$\underline{4-\mathrm{CH}_{2}} \underline{\underline{\mathrm{Cl}}} \underline{\underline{2}}$}




\begin{tabular}{|c|c|c|c|c|c|}
\hline \multirow{2}{*}{$\begin{array}{l}\text { Center } \\
\text { Number }\end{array}$} & \multirow{2}{*}{\multicolumn{2}{|c|}{$\begin{array}{l}\text { Atomic } \\
\text { Number }\end{array}$}} & \multicolumn{3}{|c|}{ Coordinates (Angstroms) } \\
\hline & & & X & $\mathrm{Y}$ & $\mathrm{Z}$ \\
\hline 1 & 6 & 0 & -2.406492 & -0.707462 & 0.003358 \\
\hline 2 & 6 & 0 & -2.406403 & 0.707563 & 0.004070 \\
\hline 3 & 46 & 0 & -0.426780 & -0.000021 & -0.001879 \\
\hline 4 & 7 & 0 & 1.368979 & 1.319927 & -0.001453 \\
\hline 5 & 6 & 0 & 2.508931 & 0.736643 & 0.002974 \\
\hline 6 & 6 & 0 & 2.508891 & -0.736654 & 0.003368 \\
\hline 7 & 7 & 0 & 1.368917 & -1.319902 & -0.001523 \\
\hline 8 & 1 & 0 & 1.427877 & -2.338369 & -0.002428 \\
\hline 9 & 1 & 0 & 3.460624 & -1.268858 & 0.007416 \\
\hline 10 & 1 & 0 & 3.460695 & 1.268798 & 0.006082 \\
\hline 11 & 1 & 0 & 1.427969 & 2.338393 & -0.003168 \\
\hline 12 & 1 & 0 & -2.637530 & -1.253037 & -0.908722 \\
\hline 13 & 1 & 0 & -2.632714 & -1.254533 & 0.915813 \\
\hline 14 & 1 & 0 & -2.637380 & 1.254122 & -0.907437 \\
\hline 15 & 1 & 0 & -2.632512 & 1.253719 & 0.917101 \\
\hline
\end{tabular}

$\begin{array}{lccc}\text { Maximum Force } & 0.000105 & 0.000450 & \text { YES } \\ \text { RMS Force } & 0.000028 & 0.000300 & \text { YES } \\ \text { Maximum Displacement } & 0.000063 & 0.001800 & \text { YES } \\ \text { RMS Displacement } & 0.000017 & 0.001200 & \text { YES }\end{array}$

Predicted change in Energy=-2.379956D-06

SCF Done: $\mathrm{E}(\mathrm{RB}+\mathrm{HF}-\mathrm{LYP})=-394.740436456$ A.U. after 8 cycles Convg $=0.5519 \mathrm{D}-08 \quad \mathrm{~S} * 2=0.0000$

\section{$\underline{\mathrm{C}}_{2} \underline{\mathrm{H}}_{4}-\mathrm{CH}_{2} \underline{\mathrm{Cl}}_{2}$}

\begin{tabular}{|c|c|c|c|c|c|}
\hline \multirow{2}{*}{$\begin{array}{l}\text { Center } \\
\text { Number }\end{array}$} & \multirow{2}{*}{$\begin{array}{l}\text { Atomic } \\
\text { Number }\end{array}$} & \multicolumn{4}{|c|}{ Coordinates (Angstroms) } \\
\hline & & $\mathrm{X}$ & $\mathrm{Y}$ & $\mathrm{Z}$ & \\
\hline 1 & 6 & 0.000000 & 0.000000 & 0.6 & 64278 \\
\hline 2 & 6 & 0.000000 & 0.000000 & -0. & 564278 \\
\hline 3 & 1 & 0.000000 & 0.923318 & -1.2 & 234575 \\
\hline 4 & 1 & 0.000000 & 0.923318 & & 34575 \\
\hline 5 & 1 & 0.000000 & -0.923318 & -1 & 234575 \\
\hline 6 & 1 & 0.000000 & -0.923318 & & 34575 \\
\hline \multicolumn{2}{|c|}{ Maximum Force } & \multicolumn{2}{|c|}{0.000169} & & YES \\
\hline RMS & Force & 0.0000 & 0.0003 & & YES \\
\hline
\end{tabular}


Maximum Displacement $\quad 0.000957 \quad 0.001800 \quad$ YES RMS Displacement $0.000541 \quad 0.001200 \quad$ YES

Predicted change in Energy=-2.914656D-07

SCF Done: $\mathrm{E}(\mathrm{RB}+\mathrm{HF}-\mathrm{LYP})=-78.6160114793 \quad$ A.U. after 6 cycles Convg $=0.6504 \mathrm{D}-08 \quad \mathrm{~S} * * 2=0.0000$

\section{$\left[4 / \mathrm{C}_{2} \underline{\mathrm{H}_{4}}\right]^{*}-\mathrm{CH}_{2} \underline{\mathrm{Cl}_{2}}$}

\begin{tabular}{|c|c|c|c|c|c|}
\hline \multirow{2}{*}{$\begin{array}{l}\text { Center } \\
\text { Number }\end{array}$} & \multirow{2}{*}{\multicolumn{2}{|c|}{$\begin{array}{l}\text { Atomic } \\
\text { Number }\end{array}$}} & \multicolumn{3}{|c|}{ Coordinates (Angstroms) } \\
\hline & & & X & Y & $\mathrm{Z}$ \\
\hline 1 & 6 & 0 & -1.878391 & -1.470304 & 0.560466 \\
\hline 2 & 6 & 0 & 1.916861 & -1.341811 & -0.840527 \\
\hline 3 & 46 & & -0.227122 & -0.285026 & -0.04605 \\
\hline 4 & 7 & 0 & 1.471285 & 0.459312 & -1.253037 \\
\hline 5 & 6 & 0 & 2.637085 & 0.361405 & -0.703991 \\
\hline 6 & 6 & 0 & 2.706887 & -0.257298 & 0.610323 \\
\hline 7 & 7 & 0 & 1.587198 & -0.479988 & 1.217566 \\
\hline 8 & 6 & 0 & -1.666031 & 2.090856 & -0.265664 \\
\hline 9 & 6 & 0 & -1.126348 & 2.322525 & 0.935275 \\
\hline 10 & 1 & 0 & -2.560863 & -0.892597 & 1.179355 \\
\hline 11 & 1 & 0 & -1.537300 & -2.396223 & 1.015691 \\
\hline 12 & 1 & & -2.624163 & -0.665 & -1.31178 \\
\hline 13 & 1 & & -1.583841 & -2.157815 & -1.47509 \\
\hline 14 & 1 & 0 & -0.156497 & 2.795346 & 1.043060 \\
\hline 15 & 1 & 0 & -1.640103 & 2.054399 & 1.851987 \\
\hline 16 & 1 & 0 & -1.170966 & 2.393197 & -1.182113 \\
\hline 17 & 1 & & -2.6515 & 1.651814 & -0.369372 \\
\hline 18 & 1 & 0 & 1.708751 & -0.926612 & 2.126250 \\
\hline 19 & 1 & 0 & 3.678698 & -0.507912 & 1.035493 \\
\hline 20 & 1 & 0 & 3.550640 & 0.724235 & -1.175078 \\
\hline 21 & 1 & 0 & 1.487397 & 0.951137 & -2.146651 \\
\hline
\end{tabular}

$\begin{array}{lccc}\text { Maximum Force } & 0.000138 & 0.000450 & \text { YES } \\ \text { RMS Force } & 0.000019 & 0.000300 & \text { YES } \\ \text { Maximum Displacement } & 0.010731 & 0.001800 & \text { NO } \\ \text { RMS Displacement } & 0.002496 & 0.001200 & \text { NO }\end{array}$

Predicted change in Energy=-9.292559D-07

SCF Done: $\mathrm{E}(\mathrm{RB}+\mathrm{HF}-\mathrm{LYP})=-473.348176844 \quad$ A.U. after 8 cycles Convg $=0.9527 \mathrm{D}-08 \quad \mathrm{~S} * * 2=0.0000$

\section{$\underline{4 \cdot \mathrm{C}_{2}} \underline{\mathrm{H}}_{4}-\mathrm{CH}_{2} \underline{\mathrm{Cl}}_{2}$}




\begin{tabular}{cccccc} 
Center & \multicolumn{3}{c}{ Atomic } & \multicolumn{4}{c}{ Coordinates (Angstroms) } \\
Number & Number & X & Y & Z \\
---------------------- \\
1 & 6 & 0 & 1.748456 & 1.542323 & 0.362541 \\
2 & 46 & 0 & 0.242612 & -0.001793 & -0.003121 \\
3 & 7 & 0 & -1.451647 & 1.108632 & 0.706827 \\
4 & 6 & 0 & -2.616415 & 0.654403 & 0.287040 \\
5 & 6 & 0 & -2.619832 & -0.646838 & -0.284355 \\
6 & 7 & 0 & -1.457680 & -1.106616 & -0.705857 \\
7 & 6 & 0 & 1.373192 & 1.755302 & -0.954249 \\
8 & 6 & 0 & 1.753686 & -1.541512 & -0.353384 \\
9 & 6 & 0 & 1.361320 & -1.754775 & 0.958663 \\
10 & 1 & 0 & -1.496589 & -2.085323 & -0.992013 \\
11 & 1 & 0 & -3.536447 & -1.228728 & -0.351025 \\
12 & 1 & 0 & -3.530720 & 1.239565 & 0.356828 \\
13 & 1 & 0 & -1.485952 & 2.087602 & 0.992689 \\
14 & 1 & 0 & 2.684257 & 1.045833 & 0.597396 \\
15 & 1 & 0 & 1.352314 & 2.170999 & 1.153632 \\
16 & 1 & 0 & 1.953284 & 1.351988 & -1.775867 \\
17 & 1 & 0 & 0.641047 & 2.510084 & -1.214442 \\
18 & 1 & 0 & 0.627455 & -2.511021 & 1.209635 \\
19 & 1 & 0 & 1.932143 & -1.352496 & 1.787255 \\
20 & 1 & 0 & 1.369436 & -2.169495 & -1.150853 \\
21 & 1 & 0 & 2.692447 & -1.044068 & -0.574004 \\
-------------------------------------------------------
\end{tabular}

$\begin{array}{lccc}\text { Maximum Force } & 0.000257 & 0.000450 & \text { YES } \\ \text { RMS Force } & 0.000080 & 0.000300 & \text { YES } \\ \text { Maximum Displacement } & 0.000331 & 0.001800 & \text { YES } \\ \text { RMS Displacement } & 0.000088 & 0.001200 & \text { YES } \\ \text { Predicted change in Energy=-9.735365D-05 } & \end{array}$

SCF Done: $\mathrm{E}(\mathrm{RB}+\mathrm{HF}-\mathrm{LYP})=-473.355306884$ A.U. after 6 cycles Convg $=0.9485 \mathrm{D}-08 \quad \mathrm{~S} * * 2=0.0000$

\section{4 -toluene}

\begin{tabular}{|c|c|c|c|c|c|}
\hline \multirow{2}{*}{$\begin{array}{l}\text { Center } \\
\text { Number }\end{array}$} & \multirow{2}{*}{\multicolumn{2}{|c|}{$\begin{array}{l}\text { Atomic } \\
\text { Number }\end{array}$}} & \multicolumn{3}{|c|}{ Coordinates (Angstroms) } \\
\hline & & & $\mathrm{X}$ & $\mathrm{Y}$ & $\mathrm{Z}$ \\
\hline 1 & 46 & 0 & 0.426268 & -0.000254 & -0.002516 \\
\hline 2 & 6 & 0 & 2.408687 & 0.706935 & 0.005591 \\
\hline 3 & 6 & 0 & 2.409305 & -0.706306 & 0.004186 \\
\hline
\end{tabular}




$\begin{array}{cccccc}4 & 1 & 0 & 2.629433 & 1.252681 & 0.919109 \\ 5 & 1 & 0 & 2.630551 & -1.253691 & 0.916597 \\ 6 & 1 & 0 & 2.635493 & 1.254517 & -0.905327 \\ 7 & 1 & 0 & 2.636522 & -1.251883 & -0.907826 \\ 8 & 7 & 0 & -1.367647 & 1.316660 & -0.001940 \\ 9 & 6 & 0 & -2.510176 & 0.735803 & 0.003744 \\ 10 & 6 & 0 & -2.510588 & -0.735031 & 0.004486 \\ 11 & 7 & 0 & -1.368430 & -1.316625 & -0.001992 \\ 12 & 1 & 0 & -1.423663 & 2.332483 & -0.003157 \\ 13 & 1 & 0 & -3.460770 & 1.268973 & 0.007615 \\ 14 & 1 & 0 & -3.461487 & -1.267644 & 0.010058 \\ 15 & 1 & 0 & -1.425223 & -2.332408 & -0.001823\end{array}$

$\begin{array}{lccc}\text { Maximum Force } & 0.000048 & 0.000450 & \text { YES } \\ \text { RMS Force } & 0.000014 & 0.000300 & \text { YES } \\ \text { Maximum Displacement } & 0.001361 & 0.001800 & \text { YES } \\ \text { RMS Displacement } & 0.000275 & 0.001200 & \text { YES } \\ \text { Predicted change in Energy=-8.277624D-08 } & \end{array}$

SCF Done: $\mathrm{E}(\mathrm{RB}+\mathrm{HF}-\mathrm{LYP})=-394.735876057$ A.U. after 12 cycles Convg $=0.8521 \mathrm{D}-08 \quad \mathrm{~S} * 2=0.0000$

\section{$\underline{\mathbf{C}}_{2} \underline{H}_{4}$ - toluene}

\begin{tabular}{cccccc} 
Center & \multicolumn{2}{c}{ Atomic } & \multicolumn{3}{c}{ Coordinates (Angstroms) } \\
Number & Number & \multicolumn{2}{c}{ X } & Y & Z \\
--------------- & \\
1 & 6 & 0 & 0.000000 & 0.000000 & 0.664278 \\
2 & 6 & 0 & 0.000000 & 0.000000 & -0.664278 \\
3 & 1 & 0 & 0.000000 & 0.923318 & -1.234575 \\
4 & 1 & 0 & 0.000000 & 0.923318 & 1.234575 \\
5 & 1 & 0 & 0.000000 & -0.923318 & -1.234575 \\
6 & 1 & 0 & 0.000000 & -0.923318 & 1.234575 \\
- & 1 & 0 &
\end{tabular}

$\begin{array}{llll}\text { Maximum Force } & 0.000414 & 0.000450 & \text { YES } \\ \text { RMS Force } & 0.000169 & 0.000300 & \text { YES } \\ \text { Maximum Displacement } & 0.001117 & 0.001800 & \text { YES } \\ \text { RMS Displacement } & 0.000635 & 0.001200 & \text { YES } \\ \text { Predicted change in Energy=-7.081362D-07 } & \end{array}$

SCF Done: $\mathrm{E}(\mathrm{RB}+\mathrm{HF}-\mathrm{LYP})=-78.6157918932$ A.U. after 6 cycles Convg $=0.6759 \mathrm{D}-08 \quad \mathrm{~S} * * 2=0.0000$ 
One imaginary frequency calculated.

\section{$\left[4 / \mathrm{C}_{2} \underline{H}_{4}\right]^{*}$ - toluene}

\begin{tabular}{|c|c|c|c|c|c|}
\hline \multirow{2}{*}{$\begin{array}{l}\text { Center } \\
\text { Number }\end{array}$} & \multirow{2}{*}{\multicolumn{2}{|c|}{$\begin{array}{l}\text { Atomic } \\
\text { Number }\end{array}$}} & \multicolumn{3}{|c|}{ Coordinates (Angstroms) } \\
\hline & & & $\mathrm{X}$ & $\mathrm{Y}$ & $\mathrm{Z}$ \\
\hline 1 & 6 & 0 & -1.290547 & 2.244567 & 1.015768 \\
\hline 2 & 6 & 0 & -1.839128 & 2.185133 & -0.197771 \\
\hline 3 & 46 & 0 & -0.196889 & -0.313355 & -0.064646 \\
\hline 4 & 7 & 0 & 1.448712 & 0.631784 & -1.195876 \\
\hline 5 & 6 & 0 & 2.620472 & 0.537789 & -0.655848 \\
\hline 6 & 6 & 0 & 2.721623 & -0.215596 & 0.580590 \\
\hline 7 & 7 & 0 & 1.610980 & -0.504736 & 1.181453 \\
\hline 8 & 6 & 0 & -1.848799 & -1.412537 & -0.875281 \\
\hline 9 & 6 & 0 & -1.784825 & -1.591568 & 0.517802 \\
\hline 10 & 1 & 0 & -0.330054 & 2.717469 & 1.185720 \\
\hline 11 & 1 & 0 & -1.787112 & 1.830763 & 1.886118 \\
\hline 12 & 1 & 0 & -1.352283 & 2.614331 & -1.066518 \\
\hline 13 & 1 & 0 & -2.807589 & 1.727170 & -0.362983 \\
\hline 14 & 1 & 0 & 1.432855 & 1.226750 & -2.020910 \\
\hline 15 & 1 & 0 & 3.511892 & 0.998211 & -1.080363 \\
\hline 16 & 1 & 0 & 3.700963 & -0.511321 & 0.954442 \\
\hline 17 & 1 & 0 & 1.744317 & -1.057895 & 2.024743 \\
\hline 18 & 1 & 0 & -2.585122 & -0.744194 & -1.311011 \\
\hline 19 & 1 & 0 & -1.487405 & -2.184664 & -1.546548 \\
\hline 20 & 1 & 0 & -2.477239 & -1.061809 & 1.166687 \\
\hline 21 & 1 & 0 & -1.396974 & -2.516541 & 0.933759 \\
\hline
\end{tabular}

$\begin{array}{lccc}\text { Maximum Force } & 0.000111 & 0.000450 & \text { YES } \\ \text { RMS Force } & 0.000039 & 0.000300 & \text { YES } \\ \text { Maximum Displacement } & 0.012522 & 0.001800 & \text { NO } \\ \text { RMS Displacement } & 0.003969 & 0.001200 & \text { NO }\end{array}$

Predicted change in Energy=-1.537298D-07

SCF Done: $\mathrm{E}(\mathrm{RB}+\mathrm{HF}-\mathrm{LYP})=-473.345095814$ A.U. after 7 cycles Convg $=0.6615 \mathrm{D}-08 \quad \mathrm{~S} * * 2=0.0000$

Two imaginary frequencies calculated.

\section{$\underline{4 \cdot \mathrm{C}_{2}} \underline{\mathrm{H}_{4}}$ - toluene}

Center Atomic Coordinates (Angstroms) 


\begin{tabular}{cccccc} 
Number & Number & $\mathrm{X}$ & $\mathrm{Y}$ & $\mathrm{Z}$ \\
\hline 1 & 46 & 0 & 0.243853 & -0.000351 & 0.000303 \\
2 & 6 & 0 & 1.355524 & -1.746258 & 0.966263 \\
3 & 6 & 0 & 1.756316 & -1.542899 & -0.344495 \\
4 & 6 & 0 & 1.752186 & 1.547315 & 0.341657 \\
5 & 6 & 0 & 1.346537 & 1.750083 & -0.967647 \\
6 & 7 & 0 & -1.449292 & 1.090789 & 0.729366 \\
7 & 6 & 0 & -2.612874 & 0.642590 & 0.299289 \\
8 & 6 & 0 & -2.613073 & -0.643519 & -0.301926 \\
9 & 7 & 0 & -1.448757 & -1.096067 & -0.725408 \\
10 & 1 & 0 & 0.617623 & -2.497421 & 1.217669 \\
11 & 1 & 0 & 1.921005 & -1.339995 & 1.795709 \\
12 & 1 & 0 & 1.375571 & -2.173875 & -1.140402 \\
13 & 1 & 0 & 2.695718 & -1.047551 & -0.563744 \\
14 & 1 & 0 & 2.694397 & 1.055433 & 0.556724 \\
15 & 1 & 0 & 1.371623 & 2.175379 & 1.139917 \\
16 & 1 & 0 & 0.604792 & 2.498384 & -1.216355 \\
17 & 1 & 0 & 1.911266 & 1.346673 & -1.798993 \\
18 & 1 & 0 & -1.485413 & 2.056762 & 1.048192 \\
19 & 1 & 0 & -3.529474 & 1.221251 & 0.385699 \\
20 & 1 & 0 & -3.530251 & -1.221273 & -0.388358 \\
21 & 1 & 0 & -1.485432 & -2.064560 & -1.036555 \\
-----------------------------------------------------------
\end{tabular}

$\begin{array}{lccc}\text { Maximum Force } & 0.000141 & 0.000450 & \text { YES } \\ \text { RMS Force } & 0.000036 & 0.000300 & \text { YES } \\ \text { Maximum Displacement } & 0.000061 & 0.001800 & \text { YES } \\ \text { RMS Displacement } & 0.000014 & 0.001200 & \text { YES }\end{array}$

Predicted change in Energy=-7.204614D-06

SCF Done: $\mathrm{E}(\mathrm{RB}+\mathrm{HF}-\mathrm{LYP})=-473.352466508$ A.U. after 9 cycles $\begin{array}{ll}\text { Convg }=0.7542 \mathrm{D}-08 & \mathrm{~S} * * 2=0.0000\end{array}$ 